\title{
Expanding Paleoindian Diet Breadth: Paleoethnobotany of Connley Cave 5, Oregon, USA
}

\author{
Katelyn N. McDonough (D, Jaime L. Kennedy, Richard L. Rosencrance, Justin A. Holcomb, \\ Dennis L. Jenkins, and Kathryn Puseman
}

\begin{abstract}
Paleoethnobotanical perspectives are essential for understanding past lifeways yet continue to be underrepresented in Paleoindian research. We present new archaeobotanical and radiocarbon data from combustion features within stratified cultural components at Connley Caves, Oregon, that reaffirm the inclusion of plants in the diet of Paleoindian groups. Botanical remains from three features in Connley Cave 5 show that people foraged for diverse dryland taxa and a narrow range of wetland plants during the summer and fall months. These data add new taxa to the known Pleistocene food economy and support the idea that groups equipped with Western Stemmed Tradition toolkits had broad, flexible diets. When viewed continentally, this work contributes to a growing body of research indicating that regionally adapted subsistence strategies were in place by at least the Younger Dryas and that some foragers in the Far West may have incorporated a wider range of plants including small seeds, leafy greens, fruits, cacti, and geophytes into their diet earlier than did Paleoindian groups elsewhere in North America. The increasing appearance of diverse and seemingly low-ranked resources in the emerging Paleoindian plant-food economy suggests the need to explore a variety of nutritional variables to explain certain aspects of early foraging behavior.
\end{abstract}

Keywords: Paleoindian, paleoethnobotany, subsistence, Great Basin, hunter-gatherers, Western Stemmed Tradition, foraging behavior

Las perspectivas paleoetnobotánicas son esenciales para comprender las formas de vida pasadas, pero siguen estando infrarrepresentadas en investigaciónes Paleoindios. Presentamos nuevos datos arqueobotánicos y de radiocarbono de características de combustión dentro de componentes culturales estratificados en las Cuevas Connley (Connley Caves), Oregon, que reafirman la inclusión de plantas en la dieta de grupos Paleoindios. Restos botánicos de tres rascos en Cueva 5 de las Cuevas Connley muestran que las personas buscaban diversos taxones de tierras secas y una gama limitada de plantas de humedales durante los meses de verano y otoño. Estos datos añaden nuevos taxones a los conocidos de la economía alimentaria del Pleistoceno y apoyan la idea de que los grupos equipados con útiles de tradiciones Western Stemmed tenían dietas amplias y flexibles. Cuando se ve a escala continental, este trabajo contribuye a un creciente cuerpo de investigación lo que indica que las estrategias de subsistencia adaptadas regionalmente estaban en su lugar al menos durante el período Younger Dryas y que algunos recolectores en el Lejano Oeste pueden haber incorporado una gama más amplia de plantas, incluidas semillas pequeñas, verduras de hoja verde, frutas, cactus, y geófitos en sus dietas antes que otros grupos Paleoindios en otras partes de Norte América. Apariciónes más frecuentes de varios recursos diversos y percibidos de ser menos importantes en la economía vegetal emergente de Paleoindio sugiere que es posible que necesitemos explorar una variedad de variables nutricionales para explicar ciertos aspectos del comportamiento de recolectores de épocas tempranos.

Palabres claves: Paleoindio, paleoetnobotánica, subsistencia, Gran Cuenca, cazadores-recolectores, Tradición de Tallo Occidental, comportamiento de forrajeo

Katelyn N. McDonough (katelynmcdonough@unr.edu, corresponding author) and Richard L. Rosencrance $\square$ Department of Anthropology, Great Basin Paleoindian Research Unit, University of Nevada, Reno, NV, USA

Jaime L. Kennedy and Dennis L. Jenkins a Museum of Natural and Cultural History, University of Oregon, Eugene, OR, USA

Justin A. Holcomb $\mathbf{a}$ Kansas Geological Survey, University of Kansas, Lawrence, KS, USA

Kathryn Puseman a Paleoscapes Archaeobotanical Services Team LLC, Bailey, CO, USA

American Antiquity 87(2), 2022, pp. 303-332

Copyright (C) The Author(s), 2022. Published by Cambridge University Press on behalf of the Society for American Archaeology. This is an Open Access article, distributed under the terms of the Creative Commons Attribution licence (http://creativecommons.org/licenses/by/4.0/), which permits unrestricted re-use, distribution, and reproduction in any medium, provided the original work is properly cited.

doi:10.1017/aaq.2021.141 
U nderstanding Pleistocene subsistence strategies is critical to developing models of mobility and to answering questions about how humans settled North America. Current models of human subsistence during the late Pleistocene ( $>11,700 \mathrm{cal} \mathrm{BP})$ in North America are predominantly derived from and biased toward faunal data. Although Paleoindian food economies undoubtedly included plants, the paucity of archaeobotanical assemblages from well-dated contexts limits our view of dietary diversity and foraging choices. Recent studies, however, have recovered fragile dietary remains from Pleistocene-aged contexts, reinforcing the need for a paleoethnobotanical perspective for understanding variability in Paleoindian lifeways.

Early models of Paleoindian land use centered on evidence from archaeological sites in the Great Plains and Southwest (Haynes 1966; Heizer and Baumhoff 1970; Martin 1973), where extinct megafaunal remains including mammoth (Mammuthus columbi) and bison (Bison antiquus) were associated with fluted-point technology (Figgins 1933; Sellards 1952). Researchers posited an adaptive strategy focusing on specialized big-game hunting and high mobility (Kelly and Todd 1988; Surovell and Waguespack 2009), with some claiming that early Paleoindian groups targeted megafauna to the point of extinction (Martin 1973). Others have challenged this interpretation (e.g., Cannon and Meltzer 2004), and rigorous debate continues regarding the importance of large mammals in Paleoindian subsistence (Grayson and Meltzer 2003; Haynes and Hutson 2013; Meltzer 2020; Waguespack 2013).

The role of plants in early Paleoindian lifeways has received significantly less attention. The skew in the archaeological discourse toward hunting over gathering is at least partially due to the dearth of Pleistocene-aged archaeobotanical assemblages, especially in temperate North America where less than $10 \%$ of Pleistocene sites with preserved subsistence material have yielded substantial (more than five botanical specimens) and unequivocal dietary plant remains (Figure 1). Our comprehensive evaluation of archaeobotanical data from Pleistocene-aged sites in North America (see Supplemental Text 1) demonstrates that current evidence for
Paleoindian plant consumption is scarce-no doubt due to several factors including preservation, sampling, and the relative importance of such resources in people's diets. The record has grown in the last two decades, however, and reliable evidence for dietary plant use is known from five sites in the American Northeast, Southeast, and Great Basin, where archaeobotanical assemblages are of sufficient size and context to confidently interpret dietary use. Botanical data from these sites confirm that some early Paleoindian groups foraged for diverse plant species and suggest that regionally adapted subsistence strategies were in place by at least the Younger Dryas (ca. 12,900-11,700 cal BP).

At the Clovis site of Shawnee Minisink, Pennsylvania, Gingerich (2011) contends that charred hawthorn fruit and hickory nuts found in hearths provide the most compelling evidence of dietary plant use and attributes those remains to opportunistic foraging near camp (but see Dent 2007). At Dust Cave, Alabama, charred botanical remains from Younger Dryas-aged components containing Quad/Beaver Lake and Dalton projectile-point technology suggest people consumed a variety of nuts (hickory [Carya sp.], black walnut [Juglans nigra], acorn [Quercus sp.], hazelnut [Corylus sp.]), berries (blackberry [Rubus sp.], and grape [Vitis sp.]) and possibly seeds (Chenopodium sp.; Hollenbach 2007). These two sites provide a view of plant use east of the Rocky Mountains indicating that late Pleistocene foragers procured nuts and fruits. In the American Great Plains, Southwest, Colorado Plateau, and California, clear evidence of plant foods is absent prior to the Early Holocene (Bement et al. 2020; Gill et al. 2021; Janetski et al. 2012; Louderback 2014; Louderback and Pavlik 2017; Rosenthal and Fitzgerald 2012; Yoder et al. 2010; see Supplemental Text 1).

Three sites in the Great Basin have reliable evidence of Pleistocene plant consumption. At Paisley Caves, Oregon, charred plants in Pleistocene-aged hearths suggest that people consumed small seeds from grasses (Poaceae), goosefoot (Chenopodium sp.), cheno-ams (Amaranthaceae), tansymustards (Descurainia sp.), phacelia (Phacelia sp.), and borages (Boraginaceae), as well as parenchymous tissue of geophytes (Kennedy 2018). Younger Dryas-aged coprolites contained amaranth 


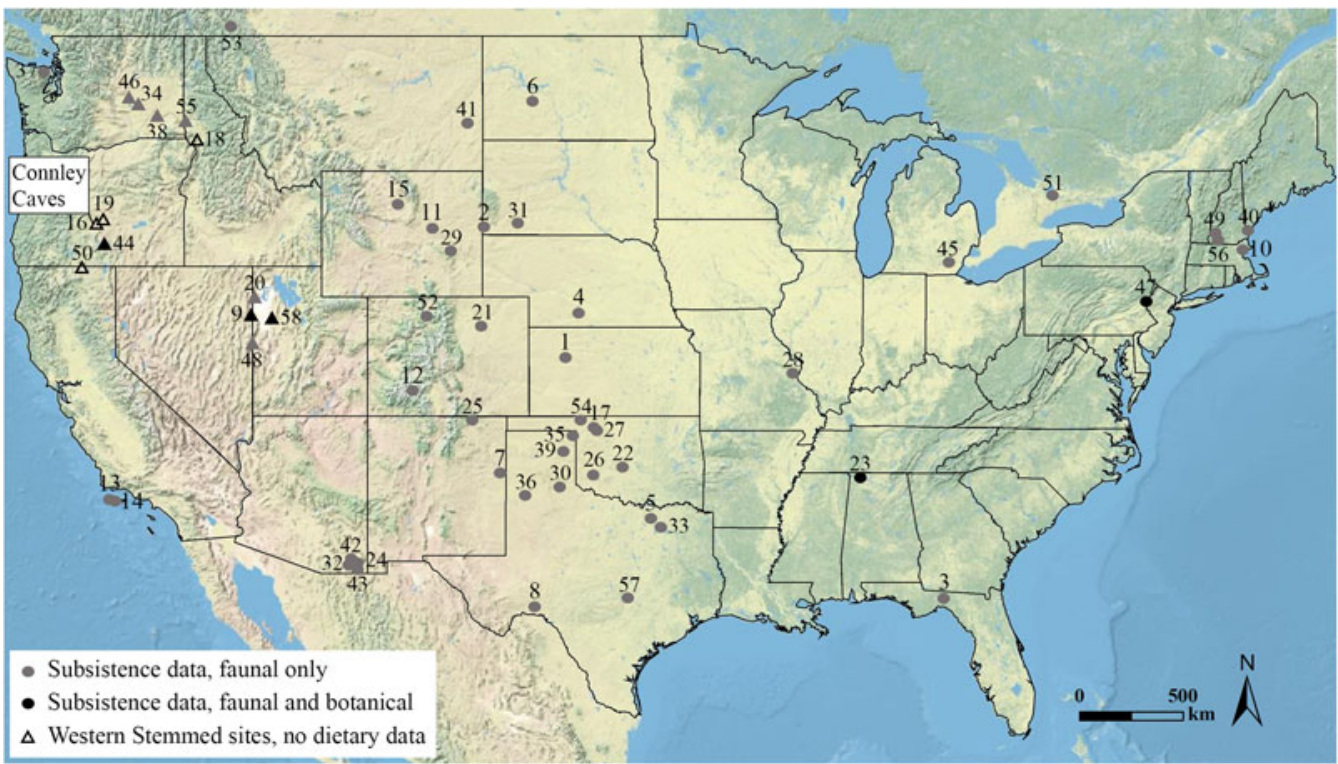

Figure 1. Map displaying location of Connley Caves and Paleoindian sites with subsistence data older than 11,700 cal BP (subsistence associations for faunal data are from Anderson et al. 2015; Gingerich and Kitchel 2015; Grayson and Meltzer 2015; Haynes and Hutson 2013; Hill 2008; Hurst et al. 2010; Kilby et al. 2021; Lothrop et al. 2016; Mackie et al. 2020; botanical data with total counts of less than five seeds were considered unreliable and excluded from the map). Sites with Western Stemmed technology are displayed as triangles, and sites with other tool technologies are displayed as circles. 1: 12 Mile Creek; 2: Agate Basin; 3: Alexon; 4: Allen; 5: Aubrey; 6: Big Black; 7: Blackwater Draw; 8: Bonfire Shelter; 9: Bonneville Estates Rockshelter; 10: Bull Brook; 11: Casper; 12: Cattle Guard; 13: Channel Islands SMI-261; 14: Channel Islands SRI-512; 15: Colby; 16: Connley Caves; 17: Cooper; 18: Cooper's Ferry; 19: Cougar Mountain Cave; 20: Danger Cave; 21: Dent; 22: Domebo; 23: Dust Cave; 24: Escapule; 25: Folsom; 26: Howard Gully; 27: Jake Bluff; 28: Kimmswick; 29: La Prele; 30: Lake Theo-Folsom; 31: Lange-Ferguson; 32: Lehner; 33: Lewisville; 34: Lind Coulee; 35: Lipscomb; 36: Lubbock Lake; 37: Manis; 38: Marmes Rockshelter; 39: Miami; 40: Michaud; 41: Mill Iron; 42: Murray Springs; 43: Naco; 44: Paisley Caves; 45: Pleasant Lake; 46: Sentinel Gap; 47: Shawnee Minisink; 48: Smith Creek Cave; 49: Tenant Swamp; 50: Tule Rockshelter; 51: Udora; 52: Upper Twin Mountain; 53: Wally's Beach; 54: Waugh; 55: Wewukiyepuh; 56: Whipple; 57: Wilson Leonard; 58: Wishbone.

(Amaranthaceae) seeds, rose fruit (Rosaceae), high frequencies of legume (Fabaceae) pollen, and common occurrences of phytoliths, indicating the regular consumption of leafy greens (Blong et al. 2020). At Bonneville Estates Rockshelter, Nevada, hearths dated between $\sim 13,000$ and 11,700 cal BP contained charred cactus (Opuntia sp.) parts and small seeds including grasses (Leymus sp., Achnatherum hymenoides, and Sporobolus sp.), goosefoot, sunflower (Asteraceae), bulrush (Schoenoplectus sp. or Bolboschoenus sp.), and mustard (Brassicaceae), all interpreted as economic (Rhode and Louderback 2007). At Wishbone, Utah, charred botanicals in a $\sim 12,300$ cal BP hearth indicate economic use of red maid (Calandrinia sp.), pitseed goosefoot (Chenopodium berlandieri), and tobacco (Nicotiana sp.; Duke et al. 2018, 2021). All three sites contain Haskett technology (Smith et al. 2020), suggesting that early stemmed-point makers had diverse diets including plants.

With the findings from Paisley Caves, Bonneville Estates Rockshelter, and Wishbone, the Great Basin region has taken center stage in the discussion of Paleoindian plant consumption. In this region, people used Western Stemmed Tradition (WST) hunting technology coeval with the Clovis Tradition (Davis et al. 2019; Jenkins et al. 2012; Shillito et al. 2020), and WST forms continued to be made through the Younger Dryas (Goebel and Keene 2014; Rosencrance 2019; Smith et al. 2020). Despite the growing Paleoindian record, WST sites still lack unequivocal evidence for the hunting of extinct megafauna (Goebel et al. 2011). Instead, current models predict that groups using WST 
technology hunted some large mammals but overall employed more diverse subsistence strategies than did groups on the Great Plains (Elston et al. 2014; Hockett 2007; Madsen 2015). The degree of resource diversity and its relationship to settlement patterns, however, are still debated (e.g., Duke and Young 2007).

Recent research at Connley Caves, a multicomponent WST site located in central Oregon, offers a new opportunity to gain a diachronic view of Paleoindian plant use. Here we present archaeobotanical data and 17 new radiocarbon ages from five combustion features spanning the Pleistocene-Holocene Transition (PHT) in Connley Cave 5 (PHT; ca. 13,000-8200 cal BP). Our objectives are to (1) characterize plant use and foraging activities at Connley Caves during the PHT, (2) consider how these data fit into the growing body of archaeobotanical data for the Pleistocene Paleoindian period more broadly, and (3) discuss implications for current settlement-subsistence models.

\section{Connley Caves}

Connley Caves are located on the traditional lands of the Klamath, Modoc, and Northern Paiute in the Fort Rock Basin of central Oregon. The Fort Rock Basin is the northwesternmost drainage system in the Great Basin, encompassing an area of $\sim 3,900 \mathrm{~km}^{2}$ bordered by the High Lava Plains (to the north), Cascade foothills (west), and woodland marshes of the Klamath Basin (south). Ridgelines divide the basin into three valleys: Silver Lake, Christmas, and Fort Rock. The site comprises at least eight rockshelters situated in the south-facing slope of Connley Hills overlooking Paulina Marsh in the Silver Lake Valley (Figure 2). Its location at the intersection of two physiographic regions and its position between the valley floor and uplands allow ready access to a variety of habitats-providing a rare look at foraging behavior in an ecotonal setting (Aikens and Jenkins 1994; Prouty 1994). The caves are $\sim 45 \mathrm{~m}$ above the valley floor $(\sim 1,356 \mathrm{~m}$ asl $)$ within a woodland belt of western juniper (Juniperus occidentalis) and a sparse understory dominated by sagebrush (Artemisia), rabbitbrush (Chrysothamnus), and grasses such as bluebunch wheatgrass (Pseudoroegneria spicata), Idaho fescue (Festuca idahoensis), and western needlegrass (Achnatherum occidentale; Bureau of Land Management 2000; Franklin and Dyrness 1988). Juniper woodland transitions into a sagebrush steppe community at the base of the Connley Hills, followed by dune formations and alkaline playa that support salt desert shrub communities of greasewood (Sarcobatus vermiculatus), shadscale (Atriplex confertifolia), and budsage (Artemisia spinescens) along the northern margin of Paulina Marsh. Emergent herbs and grass-like plants including bulrushes (e.g., Scirpus, Schoenoplectus), sedges (Carex, Cyperus), and cattail (Typha) are common throughout the marsh. Paulina Marsh's mosaic of wetland patches and meadows is a critical source of water for flora and fauna.

The site gained recognition in the archaeological community after Stephen Bedwell's excavations in the 1960s that uncovered artifacts in purported association with charcoal dating to $\sim 13,000$ cal BP (Bedwell 1970). Connley Caves also played an important role in the development of Bedwell's Western Pluvial Lakes Tradition concept, which proposed that WST groups in the western Great Basin were "tethered" to lacustrine settings. For more than 50 years, scholars have speculated about the cultural chronology of Connley Caves (Bryan 1980; Grayson 1979), with some archaeologists questioning whether they were heavily used prior to the Early Holocene (Goebel et al. 2011:493). The Archaeology Field School of the University of Oregon returned to the site between 2000 2001 and 2014-2021 to refine its chronological, ecological, and technological records (Jenkins et al. 2017; McDonough et al. 2018). Excavations in Connley Caves 4 and 5 uncovered multiple cultural components spanning the Younger Dryas through the Early Holocene (ca. 11,700-8200 cal BP; Walker et al. 2019). Here we provide the first look at the Paleoindian components recently excavated in Cave 5, which contain extensive WST lithic and osseous tool assemblages, discrete activity areas, and cultural features.

\section{Materials and Methods}

This study examines sediment samples collected between 2017 and 2019 in Connley Cave 5, 


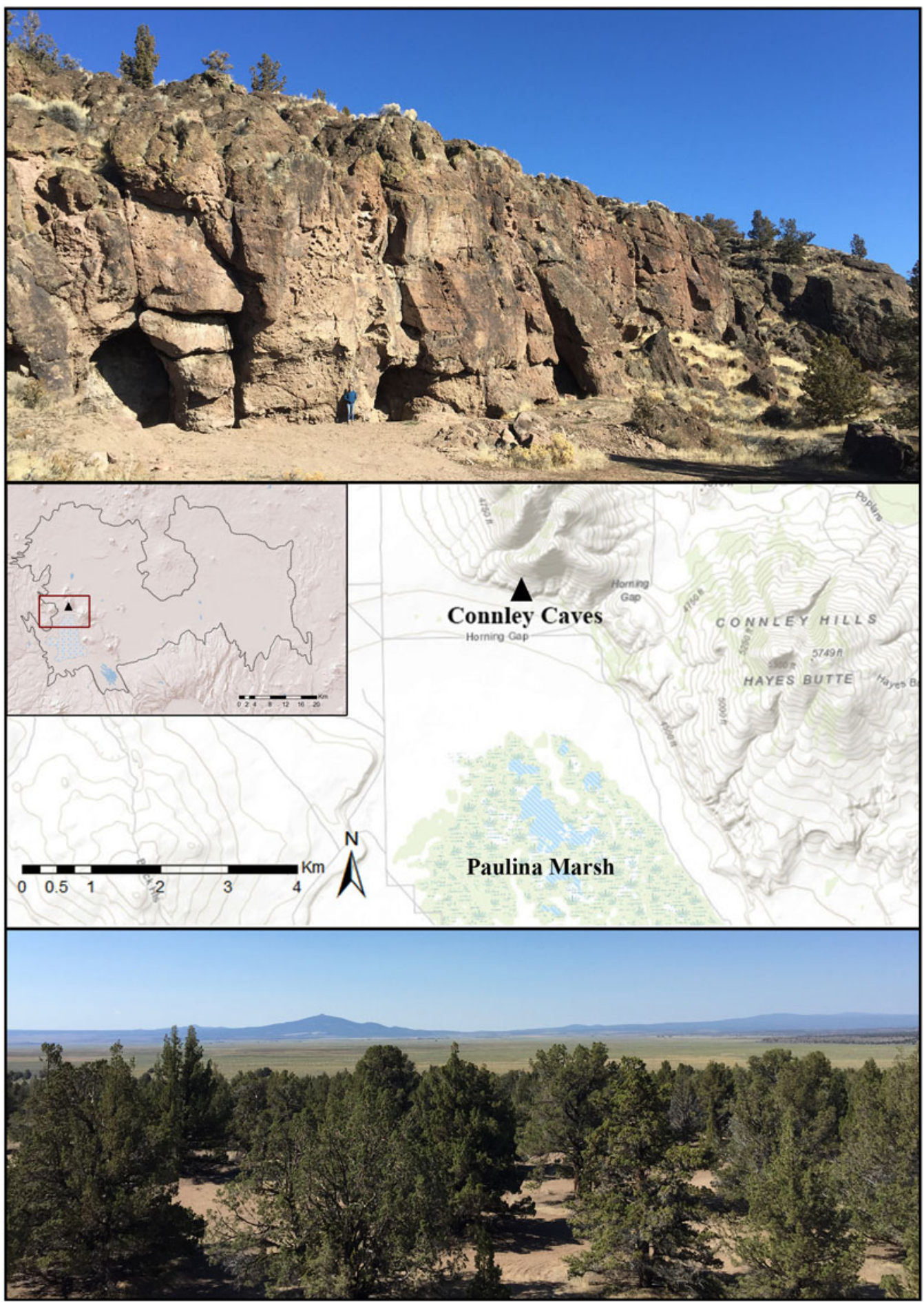

Figure 2. Top: View of Connley Caves looking north-northeast with person in blue standing above the Cave 5 excavation block. Middle: Location of the site within the Fort Rock Basin showing proximity to Paulina Marsh. Bottom: Southward view of Paulina Marsh with Hager Mountain from the top of Connley Caves (photos by Richard Rosencrance). (Color online) 
including 11 samples from inside five combustion features, two comparative samples from outside the features, and 17 control samples from an associated sediment column (Figure 3). Combustion features represent unique archaeological contexts that can inform about human behavior at the individual scale, reflecting a single event or repeated use over weeks, months, or years. Such features can take many forms in archaeological sites, and we follow the definitions outlined by Mentzer (2014) to differentiate hearths, hearth areas, and combustion areas. Supplemental Table 1 provides additional details about features and sampling methods. To establish chronological control, we submitted pieceplotted charcoal from within each combustion feature to the Pennsylvania State University AMS Radiocarbon Laboratory and Direct AMS for radiocarbon dating (Table 1).

We processed, sorted, and analyzed the bulk sediment samples at the University of Oregon Museum of Natural and Cultural History using a dry-sieving protocol adopted from Kennedy (2018:138-139). We counted each seed fragment, including identifiable and unidentifiable fragments, as one seed in the count calculation and used soil volumes to standardize samples for comparison. To examine variation among feature and column samples, we used the exploratory statistical technique of agglomerative hierarchical cluster analysis with Ward's method and made violin plots based on seed density (average number of seeds per liter) using $\mathrm{R}$ 4.0.1 ( $\mathrm{R}$ Core Team 2020). We computed richness, diversity, and evenness using Microsoft Excel. To evaluate whether plant remains should be attributed to human use or natural processes (e.g., animal activity or seed rain), we considered the abundance, spatial distribution, taphonomy, ecology, and ethnographic use of each taxon. We classified dietary association as "probable" when taxa had an abundance (total seed count) of five or greater and met at least three of the following criteria: (1) density (seeds per liter) is much higher within than outside features or the taxon is found only within features, (2) distance to the plant's habitat exceeds the range of rodent foraging and natural seed rain, (3) dietary use is ethnographically documented, and (4) there is evidence of burning or other nonrodent modification. Dietary association was "potential" if only two criteria were met, or if three were met but the seed count was less than five. Plants that do not fulfill two criteria are considered "unlikely" dietary constituents. The supplemental material contains further information on our materials and methods.

\section{Results}

\section{Stratigraphy}

Excavations at Connley Cave 5 revealed stratigraphy matching Connley Cave 4 (Jenkins et al. 2017) and clarified site-formation processes (Figures 4 and 5; Supplemental Table 2). The PHT archaeological record at Connley Cave 5 includes, from the bottom upward, windblown and colluvial deposits spanning lithostratigraphic units (LU) 2 through 5. Descriptions of each LU can be found in Supplemental Table 2. The five combustion features discussed here are preserved within LU4a, 4b, and 5. The features are distributed both horizontally and vertically throughout the Cave 5 excavation block (Tables 1 and 2). We currently interpret these features as representing separate cultural occupations, except for Features 2 and 3, which occurred at similar elevations.

\section{Features}

Feature 1 (15B-HF-2) is a bowl-shaped hearth with intact bedding and firing structure, as indicated by stratified charcoal wood fragments and ash above rubified sediment. Feature 2 (15B-HF-1) is a combustion area characterized by irregular and widespread patches of ash, dispersed charcoal, and discolored and rubified sediment with diffuse boundaries. This combustion area may represent one or multiple hearths, a rakeout, or a combination of both that was obscured via natural (e.g., wind) or cultural (e.g., foot traffic) processes. Feature $3(17-\mathrm{HF}-1)$ is a hearth area that includes both a rock-lined hearth composed of nine cobbles arranged in a circle within Unit 17 and an area of concentrated charcoal expanding out from the rock ring toward the northwest and into Unit 15B. Similarity of the fill within the feature and the contiguous combustion area to the west suggests that some of the original contents of the hearth may have been displaced 

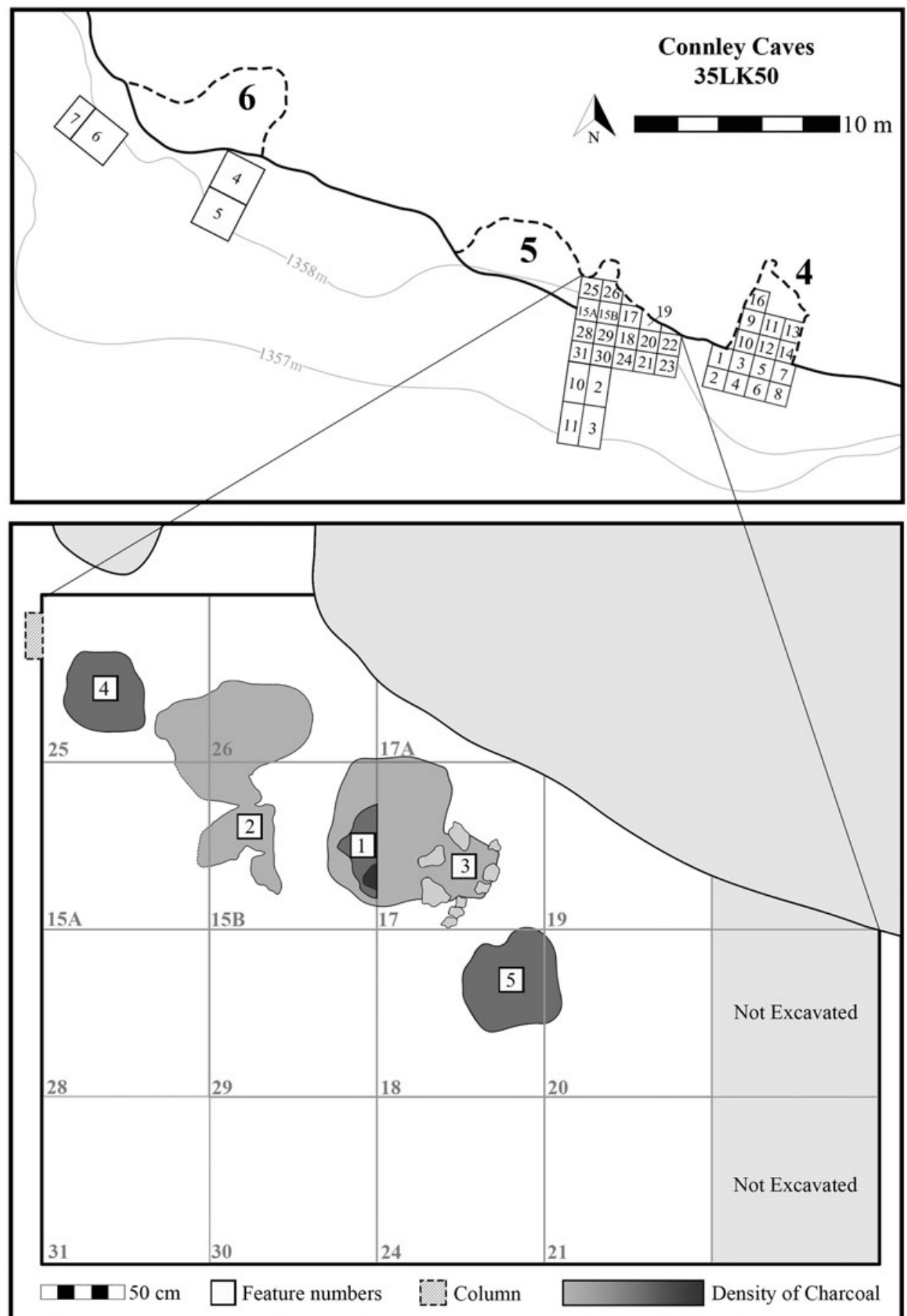

Figure 3. Planview of Connley Cave 5 (2014-2019) excavation and locations of features and column sample. Bottom image does not account for the vertical separation between features. 
Table 1. Radiocarbon Ages on Artemisia Charcoal from Cultural Features in Connley Cave 5.

\begin{tabular}{llllllr}
\hline Feature & Feature Type & Catalog Number & Lab Number & Radiocarbon Date & $95.4 \%$ Probability & LU $^{\mathrm{b}}$ \\
\hline 5 & Hearth & $5 / 18-53-7^{\mathrm{a}}$ & PSUAMS\#5246 & $9050 \pm 30$ & $10,250-10,180$ & 5 \\
5 & Hearth & $5 / 18-53-10$ & D-AMS 24523 & $9170 \pm 40$ & $10,490-10,230$ & 5 \\
5 & Hearth & $5 / 18-53-12^{\mathrm{a}, \mathrm{c}}$ & PSUAMS\#5247 & $9500 \pm 30$ & $11,070-10,590$ & 5 \\
4 & Hearth & $5 / 25-15-35^{\mathrm{a}}$ & PSUAMS\#5007 & $9985 \pm 35$ & $11,690-11,260$ & $4 \mathrm{~b}$ \\
4 & Hearth & $5 / 25-16-4$ & D-AMS 30300 & $10,010 \pm 50$ & $11,740-11,270$ & $4 \mathrm{~b}$ \\
4 & Hearth & $5 / 25-15-41^{\mathrm{c}}$ & PSUAMS\#6711 & $10,165 \pm 35$ & $11,940-11,640$ & $4 \mathrm{~b}$ \\
4 & Hearth & $5 / 25-16-64^{\mathrm{a}, \mathrm{c}}$ & PSUAMS\#5006 & $10,210 \pm 40$ & $12,010-11,740$ & $4 \mathrm{~b}$ \\
3 & Hearth Area & $5 / 17-51-95$ & D-AMS 30299 & $10,115 \pm 50$ & $11,940-11,400$ & $4 \mathrm{~b}$ \\
3 & Hearth Area & $5 / 17-51-96^{\mathrm{a}}$ & PSUAMS\#5008 & $10,120 \pm 35$ & $11,930-11,400$ & $4 \mathrm{~b}$ \\
3 & Hearth Area & $5 / 17-50-12^{\mathrm{a}}$ & PSUAMS\#5009 & $10,190 \pm 35$ & $11,970-11,730$ & $4 \mathrm{~b}$ \\
2 & Combustion Area & $5 / 15 \mathrm{~B}-31-50$ & D-AMS 30298 & $10,030 \pm 95$ & $11,880-11,240$ & $4 \mathrm{~b}$ \\
2 & Combustion Area & $5 / 15 \mathrm{~B}-32-25$ & PSUAMS\#7103 & $10,150 \pm 40$ & $11,940-11,500$ & $4 \mathrm{~b}$ \\
2 & Combustion Area & $5 / 15 \mathrm{~B}-32-38$ & PSUAMS\#6712 & $10,275 \pm 35$ & $12,440-11,820$ & $4 \mathrm{~b}$ \\
2 & Combustion Area & $5 / 15 \mathrm{~B}-32-21$ & PSUAMS\#7104 & $10,290 \pm 35$ & $12,450-11,830$ & $4 \mathrm{~b}$ \\
1 & Hearth & $5 / 15 \mathrm{~B}-36-50$ & PSUAMS\#6715 & $10,420 \pm 35$ & $12,600-12,090$ & $4 \mathrm{a}$ \\
1 & Hearth & $5 / 15 \mathrm{~B}-36-49$ & PSUAMS\#6714 & $10,490 \pm 35$ & $12,630-12,190$ & $4 \mathrm{a}$ \\
1 & Hearth & $5 / 15 \mathrm{~B}-36-51$ & PSUAMS\#6716 & $10,560 \pm 35$ & $12,690-12,480$ & $4 \mathrm{a}$ \\
\hline
\end{tabular}

${ }^{a}$ Pretreated at the Human Paleoecology and Archaeometry Lab, University of Nevada, Reno.

${ }^{\mathrm{b}}$ Lithostratigraphic unit.

${ }^{\mathrm{c}}$ Considered an outlier in this study.

outside the rock ring via wind or human activity (i.e., a rake-out). We separately analyzed samples from within (\#1781, \#1994) and outside the rock ring (\#2460) but consider them together as Feature 3 because their contents are likely to represent the same event. We, however, cannot rule out the possibility that these areas represent multiple events. Feature 4 (25-HF-2) is an intact bowlshaped hearth with a rubified base located in the center of Unit 25. Feature 5 (18-HF-1) is a shallow bowl-shaped hearth in the northeast quadrant of Unit 18.

\section{Radiocarbon Dating}

Table 1 lists new radiocarbon dates on sagebrush charcoal from the five combustion features. Supplemental Text 1 and Supplemental Table 3 contain further provenience and lab information. All the dates for Features 1, 2, and 3 internally overlap at $95.4 \%$ probability. The four dates from Feature 4 fall into two groups separated by $\sim 150$ radiocarbon years. Because Feature 4 is $\sim 20 \mathrm{~cm}$ higher in elevation than Feature 3, the younger two ages appear to date the feature. PSUAMS\#5247 is 400-300 years older than the other dates from Feature 5, suggesting it is the outlier. Because all dates are on charcoal from undisturbed contexts within cultural combustion features, bioturbation is not likely the cause of the variance. Dating inconsistencies are more likely explained by the burning of ancient wood from nearby woodrat middens as fuel, the reuse of old features later in time, or statistical scatter. We favor the first possibility because Features 4 and 5 are discrete hearths that are elevationally or stratigraphically younger in age than the other features. To summarize, the radiocarbon results indicate that Feature 1 dates to $\sim 12,500$ cal BP, Feature 2 to between $\sim 12,000$ and 11,800 cal BP, Feature 3 to $\sim 11,800$ cal BP, Feature 4 to $\sim 11,500$ cal BP, and Feature 5 to $\sim 10,200 \mathrm{cal} \mathrm{BP}$.

\section{Archaeobotanical Analysis}

Analysis of 11 sediment samples from the five combustion features yielded charred $(n=767)$ and uncharred seeds $(n=359$; see Table 2; Figures 6 and 7; Supplemental Table 4 presents individual sample counts). Represented among the charred seeds were 21 unique plant taxa, 18 of which are known edible plants included in diets of Great Basin and California Indigenous groups (Table 3; Supplemental Table 5 presents Klamath, Modoc, and Northern Paiute plant names). Other constituents include charcoal, sclerotia (mycorrhizal fungal bodies), sap, 


\section{Connley Cave 5 (35LK50) \\ Stratigraphic Framework}

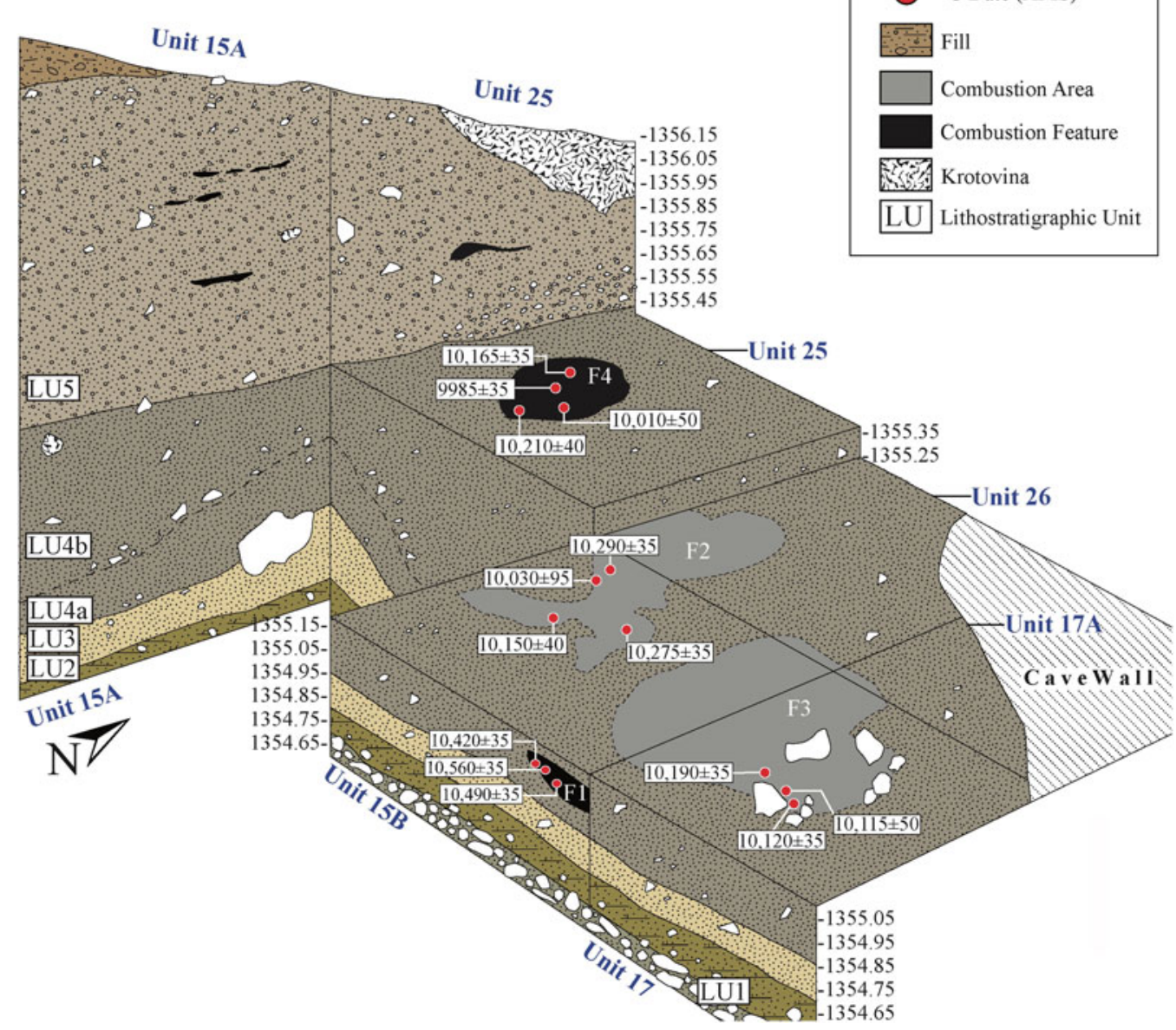

Figure 4. View of Cave 5 excavation showing Features 1-4. Feature 1 is displayed $\sim 20 \mathrm{~cm}$ farther south than its true placement (Table 1 and Figure 3 provide true provenience). (Color online)

bone, eggshell, feathers, debitage, and two boneeyed needle fragments. Three features yielded small $(<4 \mathrm{~mm})$ botanical fragments displaying characteristics consistent with processed edible tissue (PET; Stenholm 1994) that may represent plant remains prepared as food; however, further analysis is needed to confirm this association and to identify taxonomic affiliation. Thus, we report that features contain fragments of possible PET but refrain from further interpretation. The most ubiquitous taxa (present in more than $25 \%$ of the samples) from the features are goosefoot, saltbush (Atriplex sp.), peppergrass (Lepidium sp.), seepweed (Suaeda sp.), buckwheat (Eriogonum sp.), and cattail (Typha sp.). Amaranth family and cattail seeds are in the greatest abundance (total number of seeds), followed by buckwheat and sagebrush seeds. Uncharred seeds found within features are predominantly cattail (91.9\%), followed by smaller amounts of dodder (Cuscuta sp.; 2.1\%), pigweed (Amaranthus sp.; $<1 \%$ ), and unidentified fragments $(5.6 \%)$. Charred seed density is highly variable between features, ranging from an average of 1.5 seeds per liter in Feature 1 to 199.5 seeds per liter in Feature 4. Features 2, 3, and 4 have high richness and diversity values, whereas Features 1 and 5 have low richness (Table 2).

Column samples yielded 40 charred and 3 uncharred seeds (Supplemental Table 6). Plant 


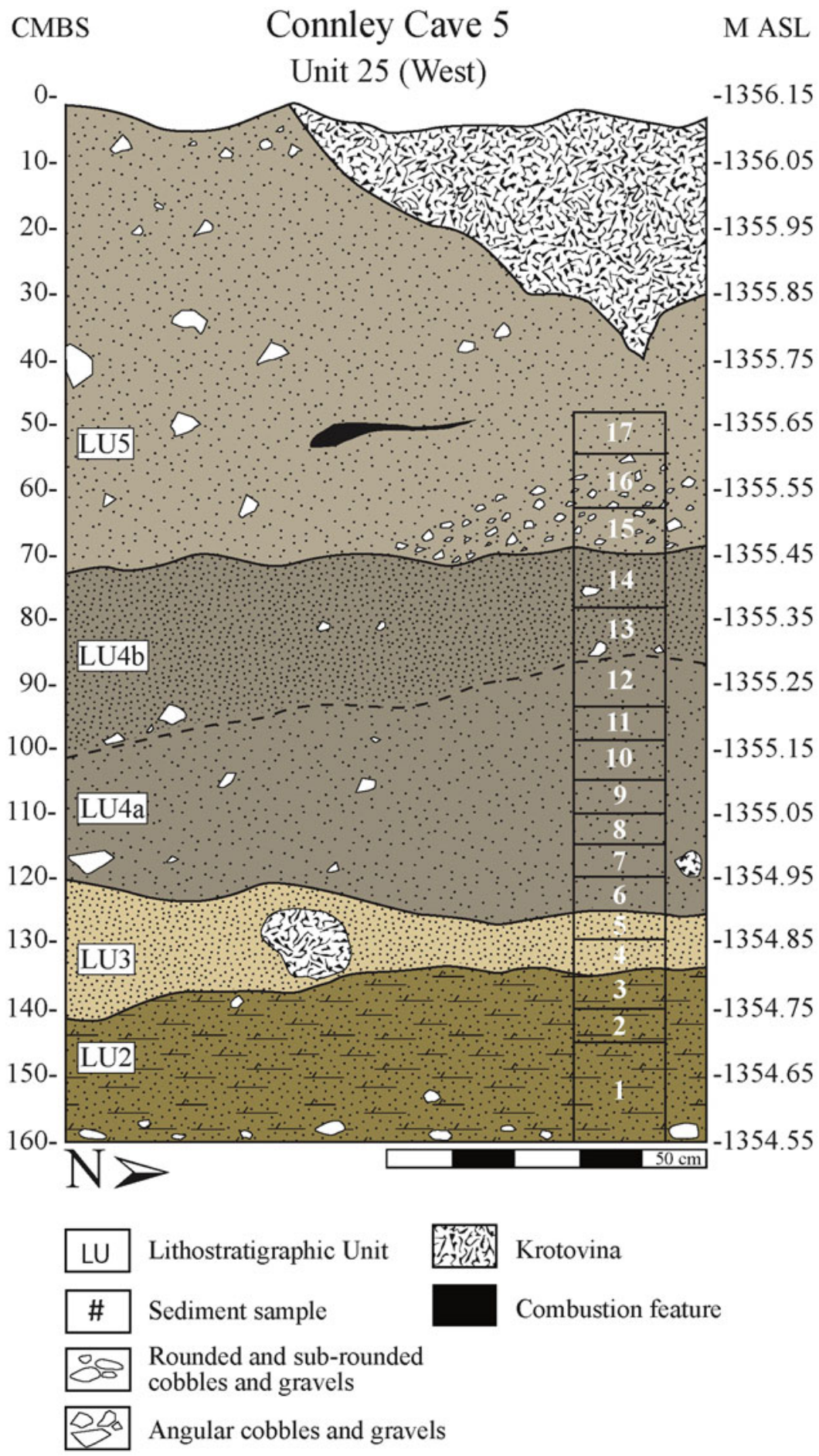

Figure 5. Stratigraphy of Unit 25's west profile with corresponding lithostratigraphic units (LUs), described in Supplemental Table 2. (Color online) 
Table 2. Feature Sample Data.

\begin{tabular}{|c|c|c|c|c|c|}
\hline Sample Data and Constituents & $\begin{array}{l}\text { Feature } 1 \\
(15 B-H F-2)\end{array}$ & $\begin{array}{l}\text { Feature } 2 \\
(15 \mathrm{~B}-\mathrm{HF}-1)\end{array}$ & $\begin{array}{l}\text { Feature } 3 \\
(17-H F-1)\end{array}$ & $\begin{array}{l}\text { Feature } 4 \\
(25-\mathrm{HF}-2)\end{array}$ & $\begin{array}{l}\text { Feature } 5 \\
(18-\mathrm{HF}-1)\end{array}$ \\
\hline Elevation (cm below $1355.95 \mathrm{~m}$ asl) & $84-93$ & $63-74$ & $65-75$ & $51-58$ & $61-63$ \\
\hline Volume (L) & 2.000 & 2.000 & 3.000 & 2.000 & 2.000 \\
\hline Total seeds & 3.000 & 579.000 & 94.000 & 445.000 & 5.000 \\
\hline Total charred seeds & 3.000 & 272.000 & 88.000 & 399.000 & 5.000 \\
\hline Charred Seed Density/1 L & 1.500 & 136.000 & 29.300 & 199.500 & 2.500 \\
\hline Shannon Index & 0.637 & 1.400 & 1.717 & 1.603 & 0.950 \\
\hline Species Richness & 2.000 & 14.000 & 14.000 & 14.000 & 3.000 \\
\hline Evenness & 0.918 & 0.531 & 0.651 & 0.607 & 0.865 \\
\hline \multicolumn{6}{|l|}{ Wetland Seeds } \\
\hline Cattail & - & 7 & - & 10 & - \\
\hline Cattail, UNCH & - & 307 & - & 23 & - \\
\hline Mare's tail & - & 1 & - & - & - \\
\hline Rush-type & - & - & - & 2 & - \\
\hline cf. Sedge & - & - & - & - & 1 \\
\hline \multicolumn{6}{|l|}{ Dryland Seeds } \\
\hline Amaranth family & 2 & 120 & 40 & 203 & 3 \\
\hline Buckwheat & - & 18 & 1 & 25 & - \\
\hline Dropseed & - & 1 & - & 4 & - \\
\hline Goosefoot & - & 14 & 5 & 5 & 1 \\
\hline Grass family & - & - & 1 & 1 & - \\
\hline cf. Mallow & - & 1 & - & - & - \\
\hline Peppergrass & - & 2 & 1 & 13 & - \\
\hline Pigweed, UNCH & - & - & 1 & - & - \\
\hline cf. Ricegrass, caryopsis & - & - & 1 & - & - \\
\hline Sagebrush & - & 1 & 1 & 12 & - \\
\hline Saltbush & - & 19 & 8 & 5 & - \\
\hline cf. Seepweed & - & 11 & 1 & 6 & - \\
\hline Sunflower family & - & - & 1 & - & - \\
\hline Whitestem blazingstar & - & - & - & 1 & - \\
\hline cf. Phacelia & - & - & 1 & - & - \\
\hline \multicolumn{6}{|l|}{ Wetland or Dryland Seeds } \\
\hline Dodder & - & - & 1 & 1 & - \\
\hline Dodder, UNCH & - & - & - & 8 & - \\
\hline Knotweed & - & - & 2 & - & - \\
\hline Spikerush & - & 1 & - & - & - \\
\hline cf. Viola & - & 2 & - & - & - \\
\hline Unidentified & 1 & 74 & 24 & 111 & - \\
\hline Unidentified, UNCH & - & - & 5 & 15 & - \\
\hline \multicolumn{6}{|l|}{ Other Botanical Material } \\
\hline Charcoal (g) & 11.85 & 25.99 & 18.76 & 6.71 & 7.24 \\
\hline Fungi sclerotia, $\mathrm{CH}$ & - & - & 8.00 & 1.00 & - \\
\hline Fungi sclerotia, UNCH & - & - & 6.00 & - & - \\
\hline cf. Processed edible tissue & - & $\mathrm{P}$ & $\mathrm{P}$ & $\mathrm{P}$ & - \\
\hline Other & - & sap & bud, nutlet & - & - \\
\hline \multicolumn{6}{|l|}{ Fauna and Artifacts } \\
\hline Bone (g) & 4.79 & 20.46 & 54.42 & 49.83 & 4.24 \\
\hline Fish vertebra & 13.00 & 108.00 & 71.00 & 427.00 & 16.00 \\
\hline Eggshell (E); feather (F) & - & $\mathrm{E}$ & $\mathrm{E}$ & $\mathrm{E} ; \mathrm{F}$ & $\mathrm{E}$ \\
\hline Debitage & 67.00 & 275.00 & 1488.00 & 121.00 & 28.00 \\
\hline Bone needle & - & 1.00 & - & 1.00 & - \\
\hline
\end{tabular}

Note: All seeds are charred unless indicated as uncharred (UNCH). Values in taxa rows are total counts unless indicated as weight $(\mathrm{g})$ or present $(\mathrm{P})$ versus absent. 


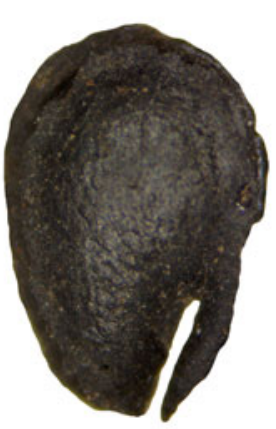

a

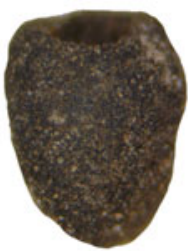

f

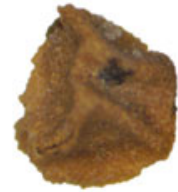

k

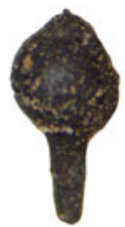

q

r

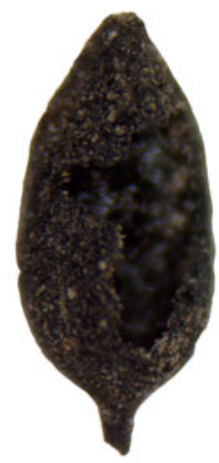

b

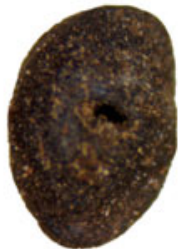

g

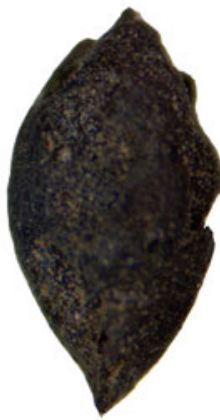

c

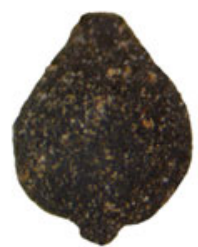

h

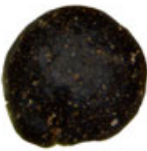

l

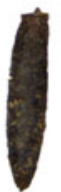

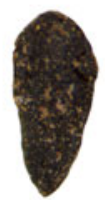

S

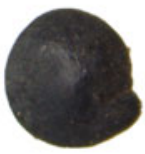

m

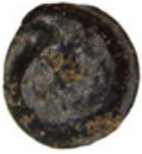

$\mathbf{n}$

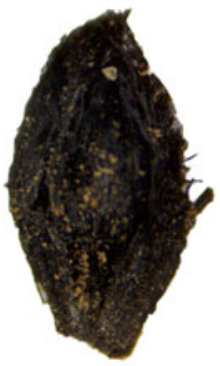

d

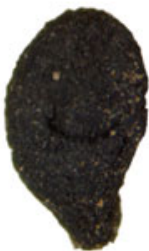

i

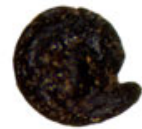

o

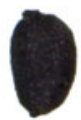

v

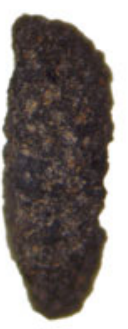

$\mathbf{e}$

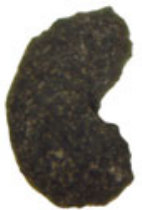

j

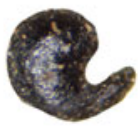

p

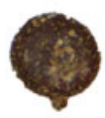

$\mathbf{W}$

\section{$1 \mathrm{~mm}$}

Figure 6. Seeds (a-c, e-w) and other macrobotanicals (d, w) recovered from combustion features: (a) peppergrass; (b) cf. sedge.; (c) knotweed family (Polygonaceae) endosperm; (d) possible bud fragment; (e) phacelia; (f) mare's tail; (g) dodder; (h) spikerush.; (i) dropseed sandgrass; (j) mallow family; (k) whitestem blazingstar; (l) amaranth family; (m) goosefoot; (n) saltbush; (o-p) cf. seepweed; (q) buckwheat; (r) cattail; (s-u) sagebrush; (v) rush; (w) sclerotia. (Color online)

taxa in the column samples include seeds of rush-type (Juncus-type), cattail, dodder, dropseed (Sporobolus sp.), goosefoot, and saltbush. Additional seeds from the grass and amaranth families could not be identified to genus. Column samples did not contain any formed tools or material resembling PET. When normalized by volume, the density of charred seeds in 


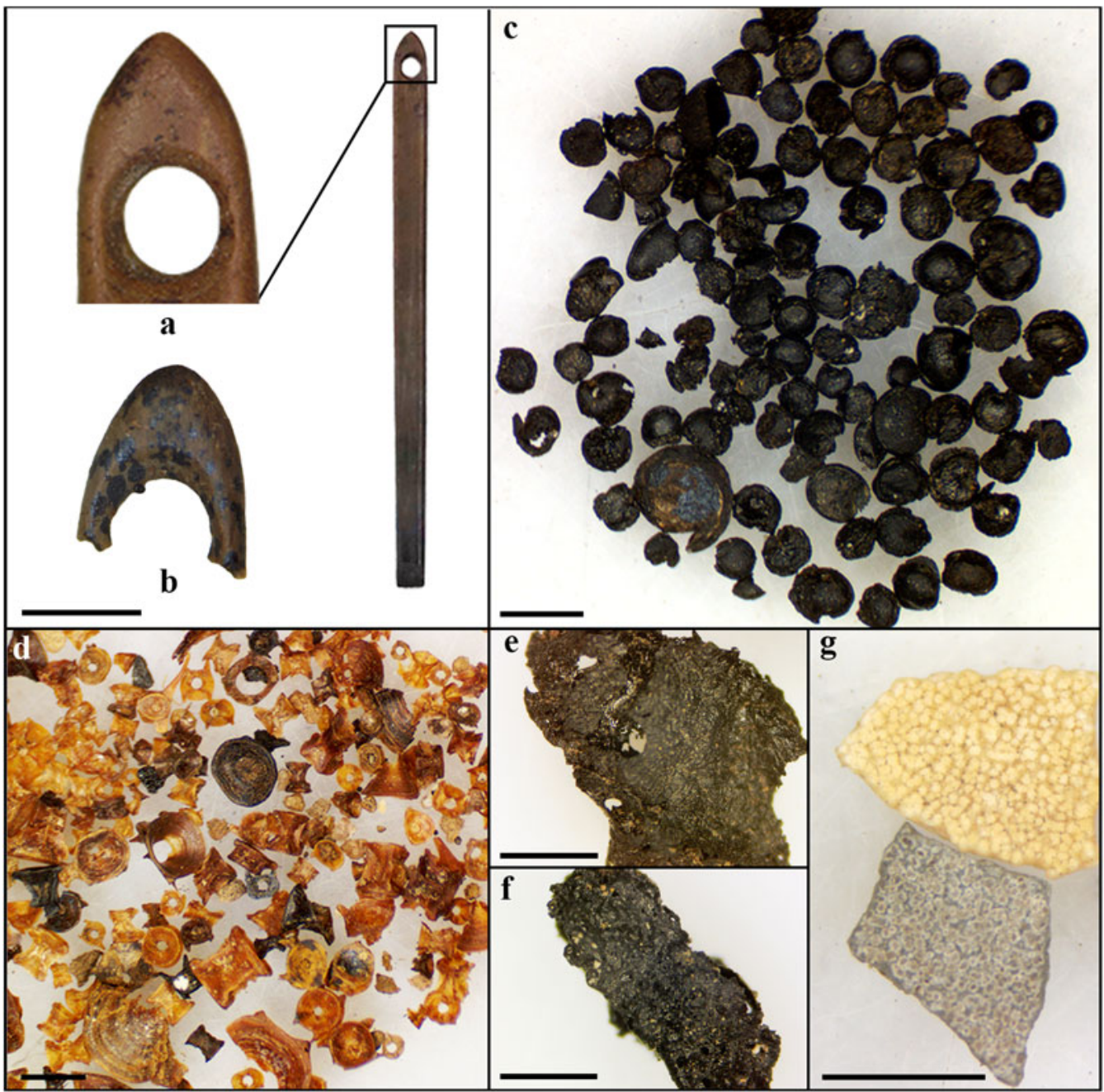

Figure 7. Other hearth constituents: (a-b) bone-eyed needle; (c) charred, fragmented Amaranthaceae seeds; (d) fish vertebrae (Feature 4); (e-f) examples of possible PET; (g) eggshell fragments (scale bars, 1 mm). (Color online)

nonfeature samples ranged from zero to $\sim 13$ per liter (excluding sample $\mathrm{C} 14$, which was less than $25 \mathrm{~cm}$ from Feature 4, and C17, which was disturbed), suggesting that natural seed deposition is characterized by low density and low diversity.

When compared, as in the violin plots in Figure 8 , there is a significant difference in the seed density of samples from Features 2, 3, and $4(M=109, S D=100)$ and samples from Feature 1, Feature 5, and the sediment column $(\mathrm{M}=4.6, \mathrm{SD}=5.8) ; t(4.9), p=0.001$. Cluster dendrograms identify Features 2 and 4 as outliers, whereas Features 1 and 5 group closely together. When analyzed with the column samples, Features
2 and 4 remain most dissimilar, whereas Features 1 and 5 group closely with the column samples (Figure 9). Feature 3 samples are dissimilar from the column samples, but less so than Features 2 and 4. Overall, this suggests that the archaeobotanical assemblages of Features 2, 3, and 4 are inconsistent with natural patterns of seed deposition at the site, whereas those from Features 1 and 5 are consistent with natural seed deposition.

\section{Feature Interpretations}

Our analysis reveals that people visiting Connley Caves during the Younger Dryas gathered and 
Table 3. Ecological and Dietary Associations of Taxa Found in Features.

\begin{tabular}{|c|c|c|c|c|c|c|c|c|c|}
\hline Family & $\begin{array}{l}\text { Genus/ } \\
\text { Species }\end{array}$ & $\begin{array}{l}\text { Common } \\
\text { Name }\end{array}$ & Parts Used ${ }^{\mathrm{a}}$ & Use & Cooking Process $^{\mathrm{a}}$ & $\mathrm{PC}^{\mathrm{b}}$ & $\begin{array}{l}\text { Seasonal } \\
\text { Availability }^{\mathrm{c}}\end{array}$ & $\begin{array}{l}\text { Dietary Assoc. } \\
\text { (Criteria Met) }\end{array}$ & $\begin{array}{l}\text { Presence at Other } \\
\text { Pleistocene-Aged WST Sites }\end{array}$ \\
\hline Amaranthaceae & Atriplex & $\begin{array}{l}\text { Saltbush/ } \\
\text { shadscale }\end{array}$ & Root, seed & $\mathrm{F}, \mathrm{M}$ & $\begin{array}{l}\text { Ground, raw, } \\
\text { parched }\end{array}$ & LOW & Fall-winter & Probable $(1,3,4)$ & $\begin{array}{l}\text { Paisley (probable), BER } \\
\text { (probable) }\end{array}$ \\
\hline Amaranthaceae & Amaranthus & Pigweed & Seed & $\mathrm{F}$ & Ground & LOW & Fall & Potential $(1,3)$ & Paisley (coprolite, probable) \\
\hline Amaranthaceae & Chenopodium & Goosefoot & Leaves, seed & $\mathrm{F}, \mathrm{M}$ & $\begin{array}{l}\text { Ground, parched, } \\
\text { raw }\end{array}$ & LOW & Fall & Probable $(1,3,4)$ & $\begin{array}{l}\text { BER (probable); Wishbone } \\
\quad \text { (potential) }\end{array}$ \\
\hline Amaranthaceae & Suaeda & Seepweed & Leaves, seed & $\mathrm{F}, \mathrm{M}$ & $\begin{array}{l}\text { Boiled, ground, } \\
\text { parched, raw }\end{array}$ & LOW & Fall & Probable $(1,3,4)$ & - \\
\hline Asteraceae & Artemisia & Sagebrush & All & $\mathrm{F}, \mathrm{M}, \mathrm{Te}, \mathrm{To}$ & $\begin{array}{l}\text { Burned, infused, } \\
\text { pounded, steeped }\end{array}$ & $\begin{array}{l}\text { LOW, } \\
\text { UPL }\end{array}$ & Summer & Probable $(1,3,4)^{\mathrm{d}}$ & BER (potential) \\
\hline Brassicaceae & Lepidium & Peppergrass & Leaves, seed & $\mathrm{B}, \mathrm{F}$ & $\begin{array}{l}\text { Boiled, dried, } \\
\text { parched, raw }\end{array}$ & - & Summer & Probable $(1,3,4)$ & - \\
\hline Plantaginaceae & Hippuris & Mare's tail & N/A & & & WET & Summer-fall & Potential $(1,2,4)$ & - \\
\hline Convolvulaceae & Cuscuta & Dodder & Stem & M & Powdered, raw & - & Year round & Unlikely (4) & - \\
\hline Cyperaceae & Cyperus & Sedge & Tuber & $\mathrm{F}$ & $\begin{array}{l}\text { Cooked, ground, } \\
\text { raw }\end{array}$ & WET & Summer-fall & Potential $(1,2,4)^{\mathrm{d}}$ & - \\
\hline Cyperaceae & Eleocharis & Spikerush & Bulbs, sap & $\mathrm{F}$ & Raw & WET & Summer-fall & Potential $(1,2,4)$ & - \\
\hline Hydrophyllaceae & Phacelia & Phacelia & Leaves, root & $\mathrm{F}, \mathrm{M}$ & Infused & - & Summer-fall & Potential $(1,3,4)$ & Paisley (potential) \\
\hline Juncaceae & Juncus-type & Rush & Seed, stem & $\mathrm{B}, \mathrm{C}, \mathrm{F}, \mathrm{Te}$ & Fermented, raw & WET & Summer & Potential $(1,3,4)^{\mathrm{d}}$ & - \\
\hline Loasaceae & $\begin{array}{l}\text { Mentzelia } \\
\quad \text { albicaulis }\end{array}$ & $\begin{array}{l}\text { Whitestem } \\
\text { blazingstar }\end{array}$ & Seed & $\mathrm{F}, \mathrm{R}, \mathrm{S}$ & $\begin{array}{l}\text { Dried, ground, } \\
\text { parched }\end{array}$ & - & Fall & Potential $(1,3,4)$ & - \\
\hline Malvaceae & Sphaeralcea & Desert mallow & Leaves, root & $\mathrm{M}, \mathrm{P}$ & $\begin{array}{l}\text { Cooked, ground, } \\
\text { infused, pounded }\end{array}$ & - & Summer & Potential $(1,3,4)$ & - \\
\hline Poaceae & $\begin{array}{l}\text { Achnatherum } \\
\text { hymenoides }\end{array}$ & Indian ricegrass & Seed & $\mathrm{F}, \mathrm{R}, \mathrm{S}$ & $\begin{array}{l}\text { Ground, parched, } \\
\text { pounded }\end{array}$ & LOW & Summer & Potential $(1,3,4)$ & $\begin{array}{l}\text { Paisley (probable), BER } \\
\text { (probable) }\end{array}$ \\
\hline Poaceae & Sporobolus & Dropseed & Seed & $\mathrm{F}$ & & LOW & Summer-fall & Potential $(1,3,4)$ & BER (probable) \\
\hline Polygonaceae & Eriogonum & Buckwheat & $\begin{array}{l}\text { Leaves, root, } \\
\text { seed, stem }\end{array}$ & $\mathrm{B}, \mathrm{F}, \mathrm{M}$ & Pounded, raw & LITH & Summer & Probable $(1,3,4)$ & - \\
\hline Polygonaceae & Polygonum & Knotweed & Seed & $\mathrm{F}$ & Ground, parched & UPL & Summer-fall & Probable $(1,3,4)$ & - \\
\hline Typhaceae & Typha & Cattail & All & $\mathrm{F}$ & $\begin{array}{l}\text { Boiled, ground, } \\
\text { parched, raw }\end{array}$ & WET & Summer-fall & Probable $(1,2,3,4)^{\mathrm{d}}$ & $\begin{array}{l}\text { Paisley (probable), BER } \\
\quad \text { (potential) }\end{array}$ \\
\hline Violaceae & Viola & Violet & - & M & $\mathrm{N} / \mathrm{a}$ & - & Summer & Potential $(1,3,4)$ & - \\
\hline
\end{tabular}


Charred Seed Density vs. Sample Type

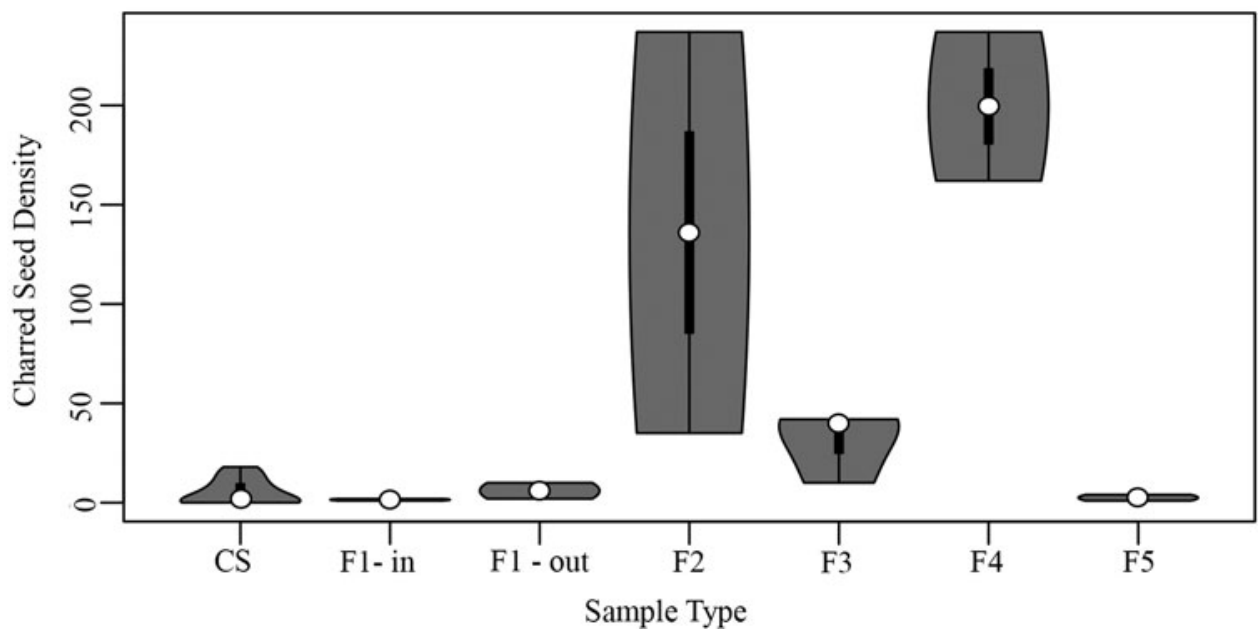

Figure 8. Total charred seed density (average number of seeds per liter) by sample type, including column samples (CS, $n=17)$; inside Feature $1(\mathrm{~F} 1$ - in, $n=2)$; outside Feature $1(\mathrm{FI}-$ out, $n=2)$; inside Feature $2(\mathrm{~F} 2, n=2)$; inside Feature 3 hearth $(F 3, n=3)$; inside Feature $4($ F4; $n=2)$; and inside Feature $5(F 5, n=2)$.

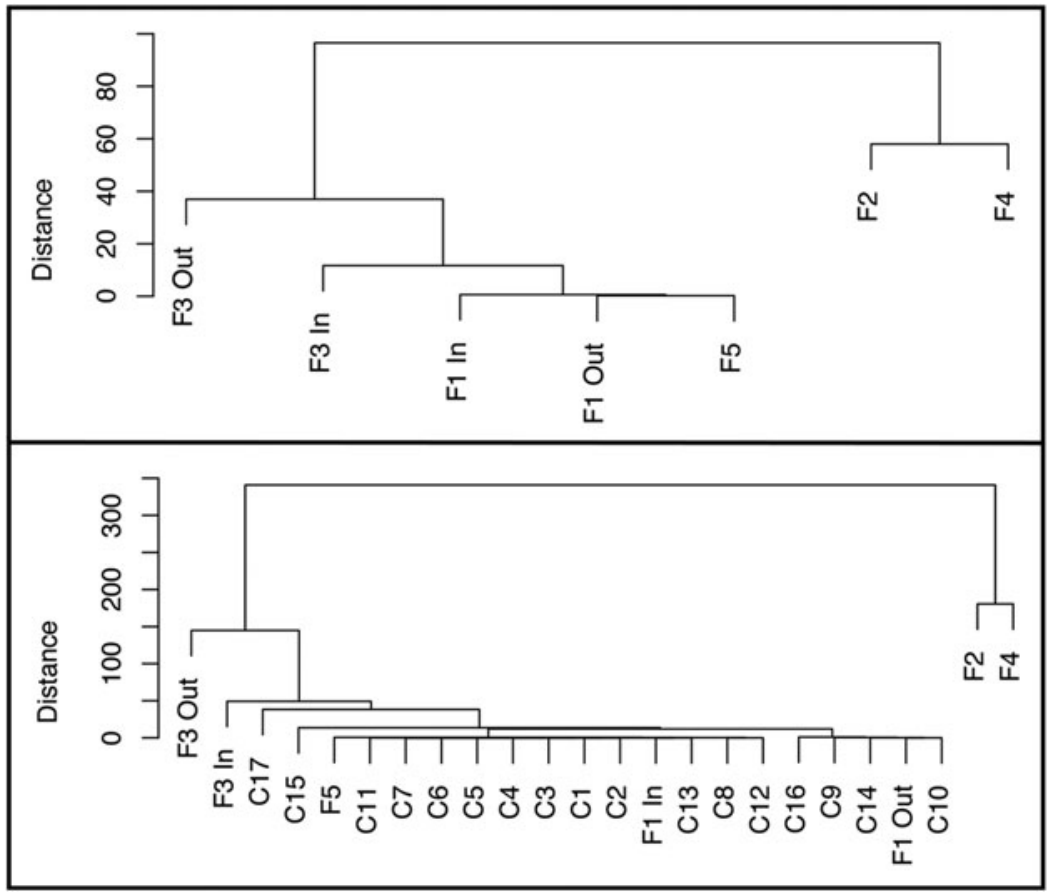

Figure 9. Cluster dendrograms of feature and column sample archaeobotanical assemblages constructed using density (average number of seeds per liter) of taxa considered "probable" and "possible" dietary constituents. 
consumed plants during multiple periods of site use. Based on our evaluative scheme, probable dietary taxa include buckwheat, cattail, goosefoot, peppergrass, saltbush, and seepweed (Table 3; Figure 10). Sagebrush seeds meet two of our criteria; however, their abundance within features is probably related to their use as fuel. Potential dietary taxa include whitestem blazingstar (Mentzelia albicaulis; partially charred), knotweed (Polygonum sp.), ricegrass (cf. Achnatherum hymenoides), phacelia (cf. Phacelia sp.), pigweed, rush, sedge, spikerush (Eleocharis sp.), violet (Viola sp.), mare's tail (Hippuris sp.), and possibly mallow (cf. Malvaceae) and sunflower (Asteraceae). Although dropseed is not commonly mentioned in the ethnographies we consulted, the seeds of this plant are consumed by Indigenous groups elsewhere in North America (Moerman 1998). Dropseed and several other taxa (e.g., cattail, rush, and spikerush) are also used in textile production and may represent other economic activities (Stevenson 1915). In this section, we interpret each feature individually with consideration of the associated nonplant materials. A summary of artifacts associated with each feature can be found in Supplemental Text 1 and Supplemental Figure 3.

\section{Feature $1(\sim 12,500$ cal BP $)$}

Feature 1 did not produce compelling evidence for dietary plant use. Densities of charred seeds inside $(n=3)$ and outside $(n=12)$ the hearth are slightly lower than the density observed in the column samples of corresponding elevation. Low density, diversity, and richness indices of plant remains in those samples suggest that they are the result of natural, rather than cultural processes. A paucity of plant remains precludes interpretation of season or foraging range; however, the lack of sagebrush seeds (the primary fuel taxa in the hearth) suggests occupation during a season other than fall. The densities of debitage, bone, and fish vertebrae are also low relative to other features.

\section{Feature $2(\sim 12,000-11,800$ cal BP)}

The botanical remains from the Feature 2 combustion area may represent multiple activities or even occupation events, therefore providing a broader view of human behavior than assemblages from the discrete hearths. Samples from Feature 2 have high seed density, diversity, and richness measurements (Table 3). Based on their abundance and ethnographic uses, the presence of buckwheat, cattail, goosefoot, saltbush, and seepweed seeds suggests subsistence activities. The seeds of dropseed, mallow, mare's tail, sagebrush, spikerush, peppergrass, and violet may also be economic but are present in low quantities. Small fragments of possible PET are present and may represent the use of geophytes or fruits. There is a greater diversity of summerseeding taxa but a greater abundance of fallseeding taxa. Because the combustion area may be a palimpsest of multiple site visits, it is unclear whether people foraged in multiple habitats during a single occupation in the late summer/early fall or during multiple visits throughout the summer and fall.

Feature 2 has the highest density of charcoal of any sample and an unusually high density of fish vertebrae. The average density of fish vertebrae in Feature 2 (54 per liter) is higher than in Features 1, 3, and 5, but slightly lower than in the corresponding column sample $(\mathrm{C} 13,88$ per liter), so it is currently equivocal whether they represent consumption. We also recovered the proximal fragment of a bone-eyed needle within this feature (Figure 7a). Overall, the constituents from Feature 2 show that people foraged for diverse plants in both wetland and dryland habitats and suggest that they may have also fished and sewed.

\section{Feature $3(\sim 11,800$ cal BP $)$}

As a hearth area, Feature 3 provides a narrower view of behavior than Feature 2 but may also represent multiple uses or events. The rock ring demonstrates some level of hearth preparation, possibly signaling prolonged feature use. The combustion area that extends outside the rock ring could be from a rake-out or, less likely, from hearth contents displaced by aeolian processes. Analysis of the contents revealed very similar constituents within and outside the rock ring, suggesting that they reflect similar activities and possibly the same event. As such, we consider them together as one hearth area.

Feature 3 contains a high density of economic plants known from other Younger Dryas sites, 


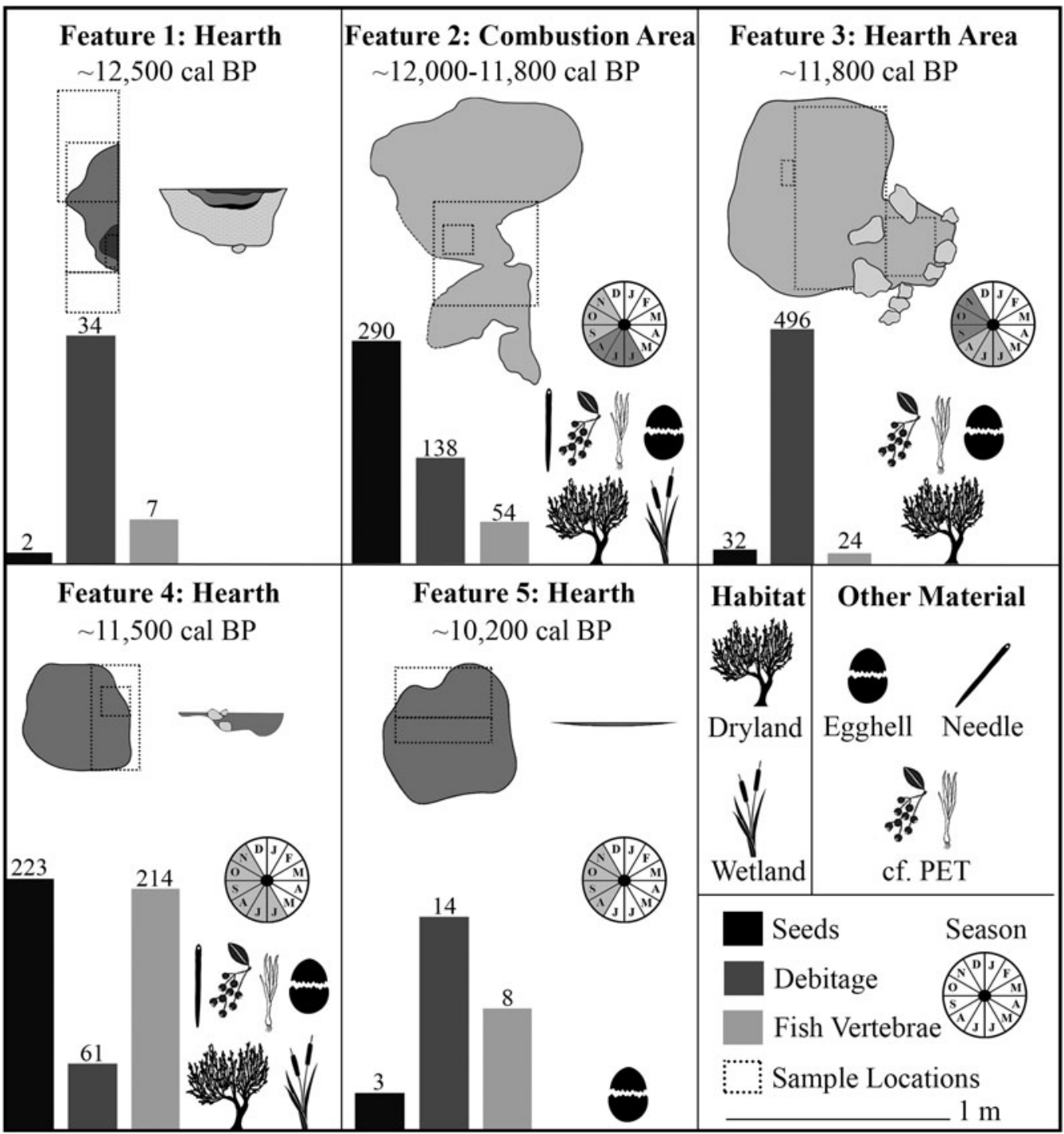

Figure 10. Summary of hearth contents (average number of seeds per liter), including all seeds recovered from features, and reflecting maximum dimensions within the feature, except for Feature 1, which was also sampled outside the hearth.

such as goosefoot and saltbush seeds. Lesser amounts of buckwheat, peppergrass, phacelia, seepweed, grass, and sunflower family seeds may also reflect subsistence. Sagebrush and dodder seeds are more likely related to medicinal plant or fuel use, rather than subsistence. Both plants are important to the Paiute and Klamath for their medicinal properties (Coville 1897; Mahar 1953; Murphey 1959; Park and Fowler 1989; Spier 1930; Train et al. 1941). Sagebrush is also a primary source of fuel at Connley
Caves and is one of the many plants that dodder parasitizes (Hitchcock and Cronquist 2018:447). One charred nutlet fragment, two charred bud fragments, and possible PET could also be subsistence related.

All the botanical taxa in Feature 3 grow in dryland contexts, primarily in lowland habitats with minor representation of lithosol (buckwheat) plant communities. All four taxa from the amaranth family seed in fall, phacelia species seed between summer and fall, and the remaining 
three taxa (buckwheat, sagebrush, and peppergrass) seed in summer. Most seeds are available closer to fall, indicating a very late summer or fall occupation. Overall seed density and diversity for Feature 3 are higher than for Features 1 and 5 and most column samples, suggesting consumption, but perhaps in a more limited capacity than is reflected in Features 2 and 4. Bone, charcoal, and debitage densities are higher in the samples collected outside the rock ring than the sample within it (Supplemental Table 4). Microdebitage is high in all samples of Feature 3 and greatest (633 pieces per liter) in the northwestern quadrant of the feature area. Taken together, the contents of Feature 3 suggest that people engaged in a variety of activities, including plant gathering in dryland habitats during the late summer or early fall.

\section{Feature $4(\sim 11,500$ cal BP $)$}

Feature 4 has the highest charred seed density ( 200 per liter) of all samples analyzed and provides further evidence of wetland and dryland foraging. Based on abundance, we consider buckwheat, cattail, peppergrass, goosefoot, saltbush, and seepweed to be economic. The botanical assemblage is dominated by seeds of the amaranth family (49\% seed total), but more than $90 \%$ of these cannot be identified beyond the family level due to high fragmentation and "popping" (see Figure 7c). This taphonomy could be the result of seed processing and parching. Additional seeds that may be subsistence related but occur in lower quantities include blazingstar, dropseed, and rush. Rush-type and blazingstar seeds are not present in any other feature samples, and the only other occurrence of dropseed is from C17. Our interpretation of dodder and sagebrush seeds in Feature 4 is the same as in Feature 3. There is a nearly even split between the number of taxa that seed in the summer versus the fall, suggesting that an occupation during late summer/early fall created Feature 4.

Feature 4 also yielded a tiny proximal fragment of a bone-eyed needle (see Figure 7b) and the lowest charcoal and highest fish vertebrae densities of any feature. The density of fish vertebrae ( 213 per liter) suggests fishing activities and lends further support for wetland use.
Overall, the low occurrence of debitage relative to the high density of charred seeds, fish vertebrae, and other faunal remains strongly suggests that Feature 4 is a cooking feature. The presence of both wetland and terrestrial plant taxa in the same discrete hearth indicates that cave occupants procured a range of resources from multiple habitats within a short period of time.

\section{Feature $5(\sim 10,200$ cal BP $)$}

Feature 5 stands out for having low amounts of seeds and charcoal. It also has the lowest bone, fish vertebrae, and debitage densities and is the only feature without eggshell. The botanical assemblage comprises three amaranth-family seeds, one sedge seed, and one grass seed. These taxa typically seed in the very late summer or fall, but their low occurrences prohibit any strong interpretations regarding patterns of seasonality or foraging zone.

\section{Discussion}

\section{Paleoindian Plant Use at Connley Caves}

Paleoethnobotanical analysis and radiocarbon dating of cultural features from stratified contexts at Connley Cave 5 confirm that people recurrently visited the site and consumed plants throughout the Younger Dryas and very earliest Holocene. The oldest (Feature 1, 12,500 cal BP) and youngest (Feature 5, 10,200 cal BP) features contained few archaeobotanical remains and may represent events in which plant foods were of little importance (e.g., logistical stopovers or hunting forays) or may reflect winter or spring visits, which are harder to detect through archaeobotanical analysis. Few plants are available in the winter months, and those that are harvested in spring are less likely to be preserved (e.g., fresh shoots, leafy greens, roots). Features 2, 3, and 4 produced strong evidence for plant use but reflect different foraging habitats and variable lengths of stay. Feature 2 contained both wetland and dryland taxa indicative of variable foraging zones, although this combustion area may be a palimpsest of multiple events. As an intact hearth, Feature 4 offers a narrower view of site use and, given the co-occurrence of high-density fish vertebrae with wetland and dryland plants, suggests that 
visitors fished and gathered plants in multiple habitats within a short interval of time. The Feature 3 hearth area produced a range of dryland plants but lacked evidence of wetland use. Representation of both wetland and dryland resources in Features 2 and 4 may reflect divisions in labor, whereas other features may reflect converging foraging goals. Environmentally, the presence of wetland plant and animal taxa suggests that Paulina Marsh was established and productive during the Younger Dryas, whereas the absence of juniper macrofossils indicates that the vegetation surrounding the caves may have been more open than it is today.

Our first look at the archaeobotanical assemblages of these five features provides important insights on human behavior during the PHT at Connley Cave 5, but we must also recognize the limitations of this study. Due to issues of taphonomy, the botanical remains in our sampled assemblage most likely reflect a fraction of original plant use (Lee 2012). Our view is likely biased toward plant taxa and parts that are more durable and have a greater opportunity to become discarded, carbonized, or both. Additionally, we examined just one area of a much larger site complex. Continued investigations in the site's other shelters and analysis of other dietary proxy data (e.g., zooarchaeological) will provide a more holistic picture of human activity and subsistence.

\section{Contribution to the Paleoindian Subsistence Record}

Archaeobotanical analyses of the assemblages from three Connley Cave 5 features reaffirm the inclusion of plants in the diet of Paleoindian groups and contribute new taxa to the known PHT food economy. Of the 19 taxa that we interpret as probably or potentially dietary, half have been found in other Younger Dryas-aged hearth features or coprolites in the Great Basin associated with WST technology (Table 3). These include seeds of cattail, dropseed sandgrass, goosefoot, phacelia, pigweed, ricegrass, rush, saltbush, sagebrush, and other members of the sunflower family. Seeds of the amaranth family appear in cultural contexts at Paisley Caves, Bonneville Estates Rockshelter, and Wishbone. Grass seeds have been interpreted as economic at both Bonneville Estates (dropseed and ricegrass) and Paisley (ricegrass). Hearths at those two sites contained other members of the mustard family, and a Paisley hearth contained phacelia. Rhode and Louderback (2007) attributed the presence of cattail seeds in hearths at Bonneville Estates to use of fluff as fire starter; however, the recovery of cattail seeds from Younger Dryas and Early Holocene coprolites at Paisley shows that dietary use should not be ruled out. Some Northern Paiute groups toast, winnow, and consume cattail seeds (Fowler 1992; Park and Fowler 1989), as did people staying at Connley Caves during the Middle and Late Holocene (McDonough 2019). In sum, archaeobotanical data from Connley Caves and other Pleistocene-aged sites indicate that amaranths, grasses, mustards, and cattail were important and perhaps even staple plant foods for WST groups living in the Great Basin region during the late Younger Dryas and earliest Holocene.

New additions to the known Pleistocene plant-food economy include buckwheat, seepweed, and peppergrass. Buckwheat and seepweed are significant economic plants to the Klamath, Paiute, and Modoc (Chamberlin 1911; Coville 1897; Fowler 1992; Kelly 1932; Park and Fowler 1989; Ray 1963; Spier 1930), and peppergrass is used by the Kawaiisu of California and other Indigenous groups throughout North America (Moerman 1998; Zigmond 1981). Buckwheat seeds are eaten parched, ground, or raw, and other parts of the plant have medicinal value (Train et al. 1941). Seepweed is also a well-known plant food that has been found in other archaeological contexts throughout the Great Basin, including Middle and Late Holocene sites in the Fort Rock Basin (Cummings 2004; Helzer 2001). Kennedy (2018) found both buckwheat and seepweed seeds in Early Holocene-aged hearths at Paisley Caves. Peppergrass is a variety of mustard that has been found in Great Basin coprolites (e.g., Hogup Cave; Fry 1976) and hearths (e.g., Hidden Cave [Rhode 2003] and Camels Back Cave [Schmitt and Madsen 2005]), but this is its first appearance in late Pleistocene features.

Other possible additions to the current record of PHT plant use include blazingstar, knotweed, mallow family, spikerush, and violet. All these 
plants have known dietary or medicinal uses; however, except for blazingstar and knotweed, many of those uses focus on the underground or leafy parts of the plant, rather than the seeds (Chamberlin 1911; Coville 1897; Kelly 1932; Murphey 1959; Park and Fowler 1989; Steward 1933; Train et al. 1941). Some of these plants, such as mallow and violet, produce relatively few seeds compared to weedy taxa (e.g., many grass and amaranth species). Because of preparation strategies and the scale of seed production, some of these seed taxa would have had less opportunity to be introduced and carbonized within hearths. Nevertheless, all these plants have been recovered from Holocene-aged archaeological contexts in the northern Great Basin (Connolly et al. 2015; Dexter 2010; Kennedy 2018; Kennedy and Smith 2016; McDonough 2019; Prouty 2004; Puseman and Yost 2011; Sanford 1983; Stenholm 1994), and low amounts of mallow $(n=2)$ and rush $(n=1)$ seeds were found in undated cooking features in Paisley Cave 5. At least one of those Cave 5 features may have been an earth oven, based on the presence of charred starchy tissue and charred seeds of the lily (Liliaceae) family (Kennedy 2018). Though they have not been directly dated, Paisley Cave 5 hearths are likely Pleistocene in age based on their stratigraphic positions. The presence of blazingstar, knotweed, mallow family, spikerush, and violet in the Connley Caves features may be related to economic use, but we consider this association as "potential" due to their low representation.

In addition to the paleoethnobotanical results, our study offers insights on other aspects of Paleoindian subsistence. Feature 4 provides the clearest evidence-including wetland plants and high density of fish vertebrae-for wetland exploitation. In the Great Basin, Blong and colleagues (2020) recovered fish remains from a $\sim 12,200$ cal BP coprolite at Paisley Caves 2, and on the Snake River Plain of Idaho the Buhl Woman's isotopic signatures suggest that she consumed anadromous fishes (Green et al. 1998; Jazwa et al. 2021). Eiselt's (1997) analysis of boli from the abdominal cavity of Burial \#2 at Spirit Cave, Nevada, identified abundant fish remains $(n=697)$ dating to 10,500 cal BP. Lastly, if the unilaterally barbed bone rod at the
Lind Coulee site in Washington and the dated bone rod from Pyramid Lake (Nevada) (Dansie and Jerrems 2005) are related to fishing (Irwin and Moody 1978), then there may be a growing corpus of evidence indicating that fish and fishing technology were important to WST groups.

The earliest evidence for plant consumption in temperate North America comes from Paisley Caves (Feature 2/6-4; dated to $11,005 \pm 30$ and $11,055 \pm 35{ }^{14} \mathrm{C}$ BP) and Shawnee Minisink (Feature 12; dating between $11,020 \pm 30$ and $\left.10,820 \pm 50{ }^{14} \mathrm{C} \mathrm{BP}\right)$. The earliest directly dated feature analyzed for plant remains at Bonneville Estates (F3.15; 10,760 \pm 70 and $10,800 \pm 60{ }^{14} \mathrm{C}$ BP) contained five charred seeds, whereas hearths dating to the mid-Younger Dryas (e.g., F4.15 dated to $10,645 \pm 45$ and F4.14 dated to $10,540 \pm 40{ }^{14} \mathrm{C}$ BP) contain higher amounts and a more diverse set of charred plant remains (Goebel et al. 2021; Rhode and Louderback 2007). Archaeobotanical data from Dust Cave, Wishbone, and other features from Paisley Caves and Bonneville Estates reflect continued plant use throughout the mid- to late Younger Dryas. Our research at Connley Caves offers a new location where people consumed plants between $\sim 12,000$ and 11,500 cal BP and adds additional taxa to the known Younger Dryas food economy.

The scarcity of groundstone technology and archaeobotanical remains from late Pleistocene sites has led some researchers to contend that plants, and small seeds specifically, did not become a significant part of the diet until the Early Holocene (Beck and Jones 1997; Fowler 1986; Jennings 1957; O'Connell et al. 1982; Rhode et al. 2006). The fact that when circumstances-for example, adequate preservation and sampling methods-allow, we find evidence of plant use prior to the appearance of formal tools associated with milling (e.g., groundstone) and parching (e.g., trays, basketry) raises the question of how people processed and consumed those resources. Plant taxa recovered from the Connley Caves can be prepared in a variety of ways, including being parched, ground, boiled, roasted, pounded, or eaten raw (Table 3). These preparation methods do not necessarily require specialized milling equipment. For instance, the seeds of the amaranth family can be "popped" 
via parching; the taphonomy of such seeds in Feature 4 (e.g., charred and split-open seed coats) suggests that people did just that. Seeds may also have been boiled in baskets or skin bags or pounded using informal stones (Coville 1897; Kelly 1932; Mahar 1953; Spier 1930). Poor organic preservation in the lower levels of Connley Cave 5 makes the recovery of perishable artifacts unlikely, but we did recover three fragments of possible expedient groundstone in association with Features 2 and 3 that are undergoing analysis for use wear and microfossils. A recent analysis of Early and Middle Holocene artifacts at Hogup Cave in Utah found that milling stones were primarily associated with starch granules from geophytes, whereas basketry yielded starches from small-seeded plant taxa (Herzog and Lawlor 2016). Herzog and Lawlor (2016:677) determined that their results "undercut the use of the presence of milling stones as a proxy for increased reliance on seeds" and concluded that more detailed studies are needed to understand the function of groundstone and textile tools. In sum, the lack of specialized milling equipment in early tool assemblages does not necessarily equate to an absence of seed use or the insignificance of plant foods, because Paleoindian groups may have processed plants using organic or expedient implements that are difficult to detect archaeologically.

Archaeobotanical assemblages from some of the oldest preserved hearths in North America suggest that plants have always been a part of people's diets; however, the range and intensity of plant gathering practices seem to have varied regionally and diachronically. Overall, the subsistence record associated with early WST tool technology, particularly Haskett projectile points, includes more diverse plant taxa than found in association with contemporaneous technocomplexes like Clovis, Vail-Debert, Bull Brook, and Dalton, which are dominated by a few taxa of wild nut mast and fruits (Gingerich and Kitchel 2015; Haynes and Hutson 2013; Hollenbach 2007). The debate continues about whether these differences were driven by variability in regional resource availability, taphonomy, recovery strategies, cultural preferences, or other factors. Most likely it is a combination of causes, which require scrutiny, review, and quantification on a continental scale. To some researchers, the archaeobotanical record east of the Rocky Mountains indicates that groups opportunistically foraged low-cost, high-return plant foods-a pattern consistent with hunting-focused lifeways (Gingerich 2011; Gingerich and Kitchel 2015; but see Hollenbach 2007). Dietary data from Paisley Caves, Bonneville Estates Rockshelter, Wishbone, and now Connley Caves indicate that intentional plant gathering was part of the larger settlement-subsistence strategy for some WST groups by at least the Younger Dryas.

\section{Implications for Settlement-Subsistence Models Based on Caloric Optimization}

Anthropologists have long been interested in forager resource-acquisition decisions, and this topic has a lively history in North American Pleistocene archaeology (Grayson and Meltzer 2003, 2015; Grayson et al. 2021; Haynes and Hutson 2013; Surovell and Waguespack 2009; Wolfe and Broughton 2020). Over the past halfcentury, human behavioral ecology (HBE) has been a productive theoretical framework for explaining past human behavior (e.g., Bettinger and Baumhoff 1982; Bird and O'Connell 2006; Codding and Bird 2015; Kelly 2013; O'Connell et al. 1982; Rhode 1999; Simms 1987; Zeanah and Simms 1999). HBE focuses on applying evolutionary theory and models of optimization to understanding human behavior within ecological contexts (Winterhalder and Smith 2000). Dietary optimization models typically use energetic returns as currency, viewing cost/ benefit relationships in terms of time/calories. Though testing these models is beyond the scope of this article, botanical data from Connley Caves and other WST sites raise questions regarding the ability of caloric optimization to explain some foraging choices. We explore those issues here.

O'Connell and colleagues (1982) were early advocates of the HBE approach in the Great Basin and were optimistic that with refined ecological data such models could be used to understand not only what happened in the past but also explain why it happened. Using a diet breadth model, they proposed that humans should have bypassed most low-ranked plant resources such as small seeds during the PHT due to the 
abundance of artiodactyl populations. Simms (1987) agreed that, based on energetic return rates, seeds should be ignored, but he also observed that such a prediction did not match regional ethnographic records. Pinson (1999) argued that risk-adverse foragers of the late Pleistocene positioned their camps in lowland ecotonal settings where lower-ranked (i.e., small game) but predictable resources could be acquired from multiple habitats. More recently, Elston and colleagues developed a comprehensive HBE-grounded model for settlementsubsistence during the PHT (Elston and Zeanah 2002; Elston et al. 2014). Integrating models of optimal foraging and sexual division of labor, they proposed that groups situated their residential camps near wetlands so that women could supplement diets with small game and riparian resources while men pursued large game. They contended that the greater prevalence and productivity of wetlands during the PHT reduced the cost of travel between patches, making it more energetically efficient for groups to move to a new patch when high-ranked resources (e.g., artiodactyls) were depleted, rather than broadening their diets to include low-ranked resources. These HBE-based models have proven very useful for generating testable expectations, but they do not make specific predictions about which plant foods would be selected and when. Although there is general agreement that Great Basin groups were never strictly big-game hunting specialists, most researchers contend that foraging goals still should have focused on highreturn resources, which would have been more abundant during the PHT.

Some researchers explain selection of lowranked resources, especially seeds, based on their storability (Simms 1985; Testart et al. 1982), and this risk-reduction strategy is evident in the Fort Rock Basin by the Middle Holocene (Jenkins 2004; Prouty 2004). Clear evidence of plant-food storage, however, has yet to be discovered in the late Pleistocene record (Smith and Barker 2017). Storability is also unable to account for the use of highly perishable foods such as leafy greens. Therefore, neither energetic maximization nor storability/risk aversion seems to explain certain foraging choices made by some Paleoindian groups. Why then, despite comprising the lowest-ranked resource class in terms of caloric return rates (Simms 1985), do small seeds recur at Paleoindian sites in the Great Basin?

We propose that people may have consumed plants to acquire essential nutrients needed for human growth and reproduction and that approaches from nutritional ecology may help explain those choices. Nutritional ecology examines the relationship between essential nutrient intake and health, recognizing that optimal health requires a range of micronutrients (e.g., vitamins and minerals) in addition to macronutrients, such as carbohydrates, fats, and proteins (Hockett 2012a; Hockett and Haws 2003; Raubenheimer and Simpson 2016). This means that foragers who consume a diverse, nutrient-rich diet would benefit from a better nutritional framework for growth, reproduction, and, therefore, population increase than groups with narrow diets. Nutritional ecology has been effectively applied in the interpretation of the Pleistocene zooarchaeological record of the Great Basin (Hockett 2007), as well as in other time periods and regions (Hockett 2012b; Hockett and Haws 2003, 2005), but not to the archaeobotanical record of the PHT in North America. Kennedy and Smith's (2016) application to the transHolocene (ca. $~ 10,000$ cal BP to contact) archaeobotanical record at LSP-1 rockshelter, Oregon, revealed correlations between nutritional diversity and population growth. Cattail and species of the amaranth and grass families from LSP-1 hearths contain a suite of essential nutrients difficult to acquire through meat alone- -highlighting the potential for nutritional ecology to help understand early plant use.

Our initial comparison of available nutritional data (Phillips et al. 2014; USDA 2019) for taxa similar to dietary constituents recovered from Connley Caves and other Paleoindian sites shows that, overall, plant foods are the best sources for carbohydrates, folate, magnesium, Vitamin A RAE, Vitamin C, potassium, calcium, and manganese (Table 4; also see discussions in Mailer and Hale 2013; Phillips et al. 2014). Each of these nutrients is vital for a properly functioning human body and its repair, growth, and reproduction. Magnesium and folate, for example, are essential during pregnancy to support embryonic 
Table 4. Comparison of Macro- and Micronutrients of Traditionally Foraged Plant and Animal Taxa.

\begin{tabular}{|c|c|c|c|c|c|c|c|c|c|c|c|c|}
\hline $\begin{array}{l}\text { Concentration } \\
\text { per } 100 \mathrm{~g}\end{array}$ & Rabbit & Bison & Elk & Grouse & Trout & $\begin{array}{l}\text { Beaked } \\
\text { Hazelnuts }\end{array}$ & Choke-cherry & $\begin{array}{l}\text { Rose } \\
\text { Hips }\end{array}$ & $\begin{array}{l}\text { Amaranth } \\
\text { Grain }\end{array}$ & $\begin{array}{l}\text { Prairie } \\
\text { Turnips }\end{array}$ & $\begin{array}{l}\text { Cattail } \\
\text { Shoots }\end{array}$ & $\begin{array}{l}\text { Lambsquarters } \\
\text { Greens }\end{array}$ \\
\hline Energy (kcal) & 114.00 & 146.000 & 111.000 & 112.000 & 119.000 & 628.00 & 156.000 & 162.000 & 102.000 & 130.000 & 25.000 & 43.000 \\
\hline Protein $(\mathrm{g})$ & 21.79 & 20.230 & 22.950 & 25.940 & 20.480 & 14.89 & 2.890 & 1.600 & 3.800 & 2.620 & 1.180 & 4.200 \\
\hline Fat $(\mathrm{g})$ & 2.32 & 7.210 & 1.450 & 0.880 & 3.460 & 52.99 & 0.980 & 0.340 & 1.580 & 0.360 & 0.000 & 0.800 \\
\hline Carbohydrate (g) & 0.00 & 0.050 & 0.000 & 0.000 & 0.000 & 22.98 & 33.880 & 38.220 & 18.690 & 35.670 & 5.140 & 7.300 \\
\hline Dietary fiber $(\mathrm{g})$ & 0.00 & 0.000 & 0.000 & 0.000 & 0.000 & 9.80 & 17.000 & 24.100 & 2.100 & 8.000 & 4.500 & 4.000 \\
\hline Calcium (mg) & 12.00 & 11.000 & 4.000 & 5.000 & 67.000 & 441.00 & 40.000 & 169.000 & 47.000 & 130.000 & 54.000 & 309.000 \\
\hline Iron (mg) & 3.20 & 2.780 & 2.760 & 0.580 & 0.700 & 3.12 & 0.400 & 1.060 & 2.100 & 1.270 & 0.910 & 1.200 \\
\hline Magnesium (mg) & 29.00 & 21.000 & 23.000 & 32.000 & 31.000 & 235.00 & 21.000 & 69.000 & 65.000 & 63.000 & 63.000 & 34.000 \\
\hline Manganese (mg) & - & - & 0.012 & 0.016 & 0.158 & 7.60 & 0.417 & 1.020 & 0.854 & 0.266 & 0.760 & 0.782 \\
\hline Potassium (mg) & 378.00 & 328.000 & 312.000 & 311.000 & 481.000 & 738.00 & 309.000 & 429.000 & 135.000 & 156.000 & 309.000 & 452.000 \\
\hline Vitamin C (mg) & 0.00 & 0.00 & 0.000 & 0.000 & 2.400 & - & 0.700 & 426.000 & - & 5.500 & 0.700 & 80.000 \\
\hline Riboflavin (mg) & 0.06 & 0.246 & - & 0.280 & 0.105 & 0.16 & 0.058 & 0.166 & 0.022 & 0.090 & 0.025 & 0.440 \\
\hline Niacin (mg) & 0.00 & 5.322 & - & 11.600 & 5.384 & 3.19 & 0.6772 & 1.300 & 0.235 & 1.071 & 0.44 & 1.200 \\
\hline Vitamin B-6 (mg) & 0.00 & 0.383 & - & 1.275 & 0.406 & 0.55 & 0.193 & 0.076 & 0.113 & 0.470 & 0.123 & 0.274 \\
\hline Folate, DFE (ug) & 0.00 & 12.000 & - & - & 12.000 & - & 10.000 & 3.000 & 22.000 & 10.000 & 0.000 & 30.000 \\
\hline $\begin{array}{l}\text { Vitamin A, RAE } \\
\text { (ug) }\end{array}$ & 0.00 & 0.000 & 0.000 & 6.000 & 19.000 & - & 2.000 & 217.000 & - & - & 1.000 & 580.000 \\
\hline Vitamin E (mg) & 0.00 & 0.190 & - & 0.730 & - & - & 0.750 & 5.840 & 0.190 & - & 0.000 & 0.000 \\
\hline Vitamin K (ug) & 0.00 & 1.200 & - & - & - & - & 29.300 & 25.900 & - & 0.000 & 22.800 & 0.000 \\
\hline
\end{tabular}

Note: Nutritional data are from the USDA National Nutrient Database for Standard Reference 28 (USDA 2019) and are given for raw food items, except for Amaranth grain, which is cooked. 
development, oocyte maturation, protein synthesis, and more; deficiencies in these and other micronutrients are linked to numerous birth complications (Fall et al. 2003; Laires et al. 2004; Ramakrishnan et al. 1999). Vitamin A is needed for bone and tissue development (Brown and Noelle 2015), vitamin C facilitates immunological and cardiovascular functions (Chambial et al. 2013), and potassium works as an electrolyte to regulate fluid balance, nerve signals, and muscle contractions (He and MacGregor 2008). This initial comparison is provocative, but nutritional data for most Paleoindian plant foods do not exist, and studies show that nutritional data for similar conventional foods are not appropriate substitutes for that of traditionally gathered wild plants, as the latter may be higher in certain healthful nutrients (Burns Kraft et al. 2008; Phillips et al. 2014). To fully understand the nutritional benefits of these and other Great Basin plant foods and to model Paleoindian plant use, more nutritional data are needed.

We do not advocate nutritional ecology instead of HBE; rather, we suggest that integrating the two approaches may be a productive way to investigate aspects of nutrition and foraging choices. Specifically, the geometric framework for nutrition may provide a useful method for integration. The geometric framework plots variables of interest (e.g., performance consequences, lifespan, reproduction, and so on) within a geometric space defined by two or more nutritional components (e.g., carbohydrates and protein) - thereby providing a powerful graphical approach for modeling how organisms prioritize competing nutritional requirements to maximize their fitness (Raubenheimer and Simpson 1993; Simpson and Raubenheimer 1993). Applications of the geometric framework to a wide range of taxa-from slime molds to locusts, mice, and humanshave demonstrated the importance of nutrient balancing (Raubenheimer and Simpson 1999, 2016; Simpson and Raubenheimer 2012), leading to calls for the use of this approach to incorporate principles from nutritional and behavioral ecologies (Morehouse et al. 2020; Raubenheimer and Simpson 2018). It therefore follows that the application of the geometric framework to the North American Paleoindian record, and to foraging data more broadly, may help explain the increasing appearance of diverse plant resources in late Pleistocene food economies. At Connley Caves, for example, the cooccurrence of charred plant remains with a lithic toolkit including specialized projectile technology may be explained by a similar division in labor to that proposed by Elston and colleagues (2014); however, perhaps rather than directly provisioning hunting activities, plant gathering diversified the diet, providing more essential nutrients and an overall better nutritional framework for growth and reproduction.

\section{Conclusions}

This project has produced one of the most robust archaeobotanical assemblages in the late Pleistocene and earliest Holocene record of North America, revealing new aspects of foraging behavior at Connley Caves and patterns in Paleoindian subsistence more broadly. We offer these four conclusions regarding the archaeobotanical assemblages from Connley Cave 5:

(1) Analysis of five combustion features shows that people recurrently visited Connley Caves during the Younger Dryas and earliest Holocene (ca. 12,500 to 10,200 cal BP). Groups harvested plants during the late summer and early fall, gathering a diverse array of dryland taxa and a narrow range of wetland plants. Fishing and sewing are also indicated by some of the features' contents.

(2) Environmental indicators, such as wetland plant taxa and fish, suggest that Paulina Marsh was established and productive in the PHT; however, marsh plants were not always the focus of food-foraging activities.

(3) The hearths from Connley Caves reaffirm and expand the diversity of plant resources in the Paleoindian food economy. These data support the proposal that WST groups in the Intermountain West had broad-based diets that incorporated resources from a variety of ecological habitats.

(4) The occurrence of seemingly low-ranked plant resources in WST sites like Connley Caves deviates from expectations of caloriebased optimization models. Our findings suggest that variables other than caloric returns may have driven some foraging 
choices, not only during the Holocene but also in the late Pleistocene, calling for the addition of a nutritional ecology perspective to HBE models.

Archaeobotany is essential for understanding past lifeways. Though limited, research has shown that plant remains can be recovered from a variety of late Pleistocene contexts, including rockshelter and open-air sites. Their analysis can reveal aspects of regional adaptations, seasonal mobility, foraging range, labor division, human health, and more. With concerted efforts to recover more micro- and macrobotanical remains and to integrate those data with other subsistence and environmental proxies, we will gain a better understanding of the behaviors long underrepresented in Paleoindian research.

Acknowledgments. We thank the Klamath Tribes and the Burns Paiute Tribe for the opportunity to conduct research at the Connley Caves, which are situated within their traditional territories. We also thank William Cannon and the Bureau of Land Management; the PLAYA Summer Lake Residency Program; the 2017-2019 Field School students and volunteers, especially Itzel Quiroz, Shelby Saper, and Kirsten Klement; and Brendan Culleton, Ted Goebel, Heather Thakar, Vaughn Bryant, and the National Plant Germplasm System. Funding was provided by the National Science Foundation (BCS-2054312 DDRI), Lakeview District BLM, University of Oregon Museum of Natural and Cultural History, Sven and Astrid Liljeblad Grant in Great Basin Studies, NAA/ Am-Arcs Student Research Grant, Arthur Hurley, and the Texas A\&M University Department of Anthropology and Center for the Study of the First Americans. We are grateful to Chantel Saban for providing the Spanish abstract translation. We thank Bryan Hockett and two anonymous reviewers for their comments, which improved this article.

Data Availability Statement. All data presented in this article are available in the supplemental material. Artifact and botanical assemblages are housed at the University of Oregon Museum of Natural and Cultural History.

Supplemental Material. For supplemental material accompanying this article, visit https://10.1017/aaq.2021.141.

Supplemental Text 1. Review of Terminal Pleistocene Archaeobotanical Data in Temperate North America and Details on Materials and Methods.

Supplemental Table 1. Feature Proveniences and Descriptions.

Supplemental Table 2. Description of Lithostratigraphic Units (LU) in Connley Cave 5.

Supplemental Table 3. Provenience and Radiocarbon Results of Feature Charcoal Samples.
Supplemental Table 4. Quantity, Density, and Diversity of Taxa Found in Connley Caves Feature Samples.

Supplemental Table 5. Names of Taxa Found in Connley Caves Features.

Supplemental Table 6. Quantity of Taxa Found in Connley Caves Column Samples.

\section{References Cited}

Aikens, C. Melvin, and Dennis L. Jenkins (editors)

1994 Archaeological Researches in the Northern Great Basin: Fort Rock Archaeology since Cressman. Anthropological Papers 50. Department of Anthropology, University of Oregon, and State Museum of Anthropology, Eugene.

Anderson, David G., Ashley M. Smallwood, and D. Shane Miller

2015 Pleistocene Human Settlement in the Southeastern United States: Current Evidence and Future Directions. PaleoAmerica 1:7-51.

Beck, Charlotte, and George T. Jones

1997 The Terminal Pleistocene/Early Holocene Archaeology of the Great Basin. World Archaeology 11:161236.

Bedwell, Stephen F.

1970 Prehistory and Environment of the Pluvial Fort Rock Lake Area of South Central Oregon. PhD dissertation, Department of Anthropology, University of Oregon, Eugene.

Bement, Leland C., Richard R. Drass, Linda Scott Cummings, and Dakota Larrick

2020 Breaking a Preservation Barrier: Recovery of Charred Seeds in a 10,270 Year-Old Hearth on the High Plains of North America. PaleoAmerica 7:68-75.

Bettinger, Robert L., and Martin A. Baumhoff

1982 The Numic Spread: Great Basin Cultures in Competition. American Antiquity 47:485-503.

Bird, Douglas W., and James F. O'Connell

2006 Behavioral Ecology and Archaeology. Journal of Archaeological Research 14:143-188.

Blong, John C., Martin E. Adams, Gabriel Sanchez, Dennis L. Jenkins, Ian D. Bull, and Lisa-Marie Shillito

2020 Younger Dryas and Early Holocene Subsistence in the Northern Great Basin: Multiproxy Analysis of Coprolites from the Paisley Caves, Oregon, USA. Archaeological and Anthropological Sciences 12(9): $1-29$.

Brown, Chrysothemis C., and Randolph J. Noelle

2015 Seeing through the Dark: New Insights into the Immune Regulatory Functions of Vitamin A. European Journal of Immunology 45:1287-1295.

Bryan, Alan L.

1980 The Stemmed Point Tradition: An Early Technological Tradition in Western North America. In Anthropological Papers in Memory of Earl H. Swanson Jr., edited by Lucille B. Harten, Claude N. Warren, and Donald R. Tuohy, pp. 77-107. Idaho State Museum of Natural History, Pocatello.

Bureau of Land Management, Lakeview District

2000 Areas of Critical Environmental Concern Nomination Analysis Report for the Lakeview Resource Area Resource Management Plan. Bureau of Land Management, Lakeview, Oregon. 
Burns Kraft, Tristan F., Moul Dey, Randy B. Rogers, David M. Ribnicky, David M. Gipp, William T. Cefalu, Ilya Raskin, and Mary Ann Lila

2008 Phytochemical Composition and Metabolic Performance-Enhancing Activity of Dietary Berries Traditionally Used by Native North Americans. Journal of Agricultural and Food Chemistry 56:654-660.

Cannon, Michael D., and David J. Meltzer

2004 Early Paleoindian Foraging: Examining the Faunal Evidence for Large Mammal Specialization and Regional Variability in Prey Choice. Quaternary Science Reviews 23:1955-1987.

Chamberlin, Ralph Vary

1911 The Ethno-Botany of the Gosiute Indians of Utah. Memoirs of the American Anthropological Association Vol. 2, No. 5. New Era Printing Company, Lancaster, Pennsylvania.

Chambial, Shailja, Shailendra Dwivedi, Kamla Kant Shukla, Placheril J. John, and Praveen Sharma

2013 Vitamin C in Disease Prevention and Cure: An Overview. Indian Journal of Clinical Biochemistry 28(4):314-328.

Codding, Brian F., and Douglas W. Bird

2015 Behavioral Ecology and the Future of Archaeological Science. Journal of Archaeological Science 56:9-20.

Connolly, Thomas J., Chris L. Ruiz, Dennis L. Jenkins, and Douglas Deur

2015 This Place Is Home: Exploring Heritage and Community of the Klamath Tribes at Beatty Curve Site (35LK95). Reports 2014-000. University of Oregon Museum of Natural and Cultural History, Eugene.

Coville, Frederick V.

1897 Notes on the Plants Used by the Klamath Indians of Oregon. Contributions from the U.S. National Herbarium Vol. 5, No. 2. Government Printing Office, Washington, DC.

Cummings, Linda Scott

2004 Great Basin Paleoethnobotany. In People and Plants in Ancient Western North America, edited by Paul E. Minnis, pp. 205-277. Smithsonian Institution, Washington, DC.

Dansie, Amy J., and Jerry Jerrems

2005 Lahontan Chronology and Early Human Occupation in the Western Great Basin: A New Look at Old Collections. In New Perspectives on the First Americans, edited by Bradley T. Lepper and Robson Bonnichsen, pp. 55-63. Center for the Study of the First Americans, Texas A\&M University, College Station.

Davis, Loren G., David B. Madsen, Lorena Becerra-Valdivia, Thomas Higham, David A. Sisson, Sarah M. Skinner, Daniel Stueber, et al.

2019 Late Upper Paleolithic Occupation at Cooper's Ferry, Idaho, USA, 16,000 Years Ago. Science 365:891-897.

Dent, R. Joseph

2007 Seed Collecting and Fishing at the Shawnee Minisink Paleoindian Site: Everyday Life in the Late Pleistocene. In Foragers of the Terminal Pleistocene in North America, edited by Renee B. Walker and Boyce N. Driskell, pp. 116-132. University of Nebraska Press, Lincoln.

Dexter, Jaime

2010 Plant Exploitation in Arid Upland Environments: Quantitative Data from the Erin's Cave Archaeological Site, Southeast Oregon. Master's thesis, Interdisiplinary Studies, University of Oregon, Eugene.
Duke, Daron, Sarah K. Rice, Craig D. Young, and Ryan Byerly

2018 The Playas Archaeological Inventory: 6,914 Acres on the Utah Test and Training Range Including Portions of the West Distal Delta of the Old River Bed and Test Excavations at the Wishbone Site (42TO6384), Tooele County, Utah. Report prepared for the Center for Integrated Research on the Environment, University of Montana, and Hill Air Force Base. Far Western Anthropological Research Group, Henderson, Nevada.

Duke, Daron, Eric Wohlgemuth, Karen R. Adams, Angela Armstrong-Ingram, Sarah K. Rice, and D. Craig Young

2021 Earliest Evidence for Human Use of Tobacco in the Pleistocene Americas. Nature Human Behaviour 2021. DOI:10.1038/s41562-021-01202-9.

Duke, Daron G., and D. Craig Young

2007 Episodic Permanence in Paleoarchaic Basin Selection and Settlement. In Paleoindian or Paleoarchaic? Great Basin Human Ecology at the Pleistocene/Holocene Transition, edited by Kelly E. Graf and Dave N. Schmitt, pp. 123-138. University of Utah Press, Salt Lake City.

Eiselt, B. Sunday

1997 Fish Remains from the Spirit Cave Paleofecal Material: 9,400 Year Old Evidence for Great Basin Utilization of Small Fishes. Nevada Historical Society Quarterly 40(1):117-139.

Elston, Robert G., and David W. Zeanah

2002 Thinking outside the Box: A New Perspective on Diet Breadth and Sexual Division of Labor in the Prearchaic Great Basin. World Archaeology 34:103-130.

Elston, Robert G., David W. Zeanah, and Brian F. Codding

2014 Living outside the Box: An Updated Perspective on Diet Breadth and Sexual Division of Labor in the Prearchaic Great Basin. Quaternary International 352:200-211.

Fall, Caroline H. D., Chittaranjan S. Yajnik, Shobha Rao, Anna A. Davies, Nick Brown, and Hannah J. W. Farrant

2003 Micronutrients and Fetal Growth. Journal of Nutrition 133:1747S-1756S.

Figgins, Jesse D.

1933 A Further Contribution to the Antiquity of Man in America, Vol. 12, No. 2. Colorado Museum of Natural History, Denver.

Fowler, Catherine S.

1986 Subsistence. In Great Basin, edited by Warren L. D’Azevedo, pp. 64-97. Handbook of North American Indians, Vol. 11, William C. Sturtevant, general editor. Smithsonian Institution, Washington, DC.

1992 In the Shadow of Fox Peak: An Ethnography of the Cattail-Eater Northern Paiute People of the Stillwater Marsh. Cultural Resource Series 5. US Department of the Interior Fish and Wildlife Service. US Government Printing Office, Washington, DC.

Franklin, Jerry F., and Christen T. Dyrness

1988 Natural Vegetation of Oregon and Washington. Oregon State University Press, Corvallis.

Fry, Gary F.

1976 Analysis of Prehistoric Coprolites from Utah. Anthropological Papers 97. University of Utah Press, Salt Lake City.

Gill, Kristina M., Todd J. Braje, Kevin Smith, and Jon M. Erlandson

2021 Earliest Evidence for Geophyte Use in North America: 11,500-Year-Old Archaeobotanical Remains from California's Santarosae Island. American Antiquity 86:625-637. 
Gingerich, Joseph A. M.

2011 Down to Seeds and Stones: A New Look at the Subsistence Remains from Shawnee-Minisink. American Antiquity 76:127-144.

Gingerich, Joseph A. M., and Nathaniel R. Kitchel

2015 Early Paleoindian Subsistence Strategies in Eastern North America: A Continuation of the Clovis Tradition? Or Evidence of Regional Adaptations. In Clovis: On the Edge of a New Understanding, edited by Ashley M. Smallwood and Thomas A. Jennings, pp. 297-318. Texas A\&M University Press, College Station.

Goebel, Ted, Bryan Hockett, Kenneth D. Adams, David Rhode, and Kelly Graf

2011 Climate, Environment, and Humans in North America's Great Basin during the Younger Dryas, 12,900 11,600 Calendar Years Ago. Quaternary International 242:479-501.

Goebel, Ted, Bryan Hockett, David Rhode, and Kelly Graf 2021 Prehistoric Human Response to Climate Change in the Bonneville Basin, Western North America: The Bonneville Estates Rockshelter Radiocarbon Chronology. Quaternary Science Reviews 250:106930.

Goebel, Ted, and Joshua L. Keene

2014 Are Great Basin Stemmed Points as Old as Clovis in the Intermountain West? A Review of the Geochronological Evidence. In Archaeology for All Times: Papers in Honor of Don D. Fowler, edited by Joel Janetski and Nancy Parezo, pp. 35-60. University of Utah Press, Salt Lake City.

Grayson, Donald K.

1979 Mount Mazama, Climatic Change, and Fort Rock Basin Archaeofaunas. In Volcanic Activity and Human Ecology, edited by Payson D. Sheets and Donald K. Grayson, pp. 427-457. Academic Press, New York.

Grayson, Donald K., and David J. Meltzer

2003 A Requiem for North American Overkill. Journal of Archaeological Science 30:585-593.

2015 Revisiting Paleoindian Exploitation of Extinct North American Mammals. Journal of Archaeological Science 56:177-193.

Grayson, Donald K., David J. Meltzer, and Ryan P. Breslawski 2021 Overkill and the North American Archaeological Record-Not Guilty by Association? A Comment on Wolfe and Broughton (2020). Journal of Archaeological Science 128:105312.

Green, Thomas J., Bruce Cochran, Todd W. Fenton, James C. Woods, Gene L. Titmus, Larry Tieszen, Mary Anne Davis, and Susanne J. Miller

1998 The Buhl Burial: A Paleoindian Woman from Southern Idaho. American Antiquity 63:437-456.

Haynes, C. Vance, Jr.

1966 Elephant-Hunting in North America. Scientific American 214(6):104-115.

Haynes, Gary, and Jarod M. Hutson

2013 Clovis-Era Subsistence: Regional Variability, Continental Patterning. In Paleoamerican Odyssey, edited by Kelly E. Graf, Caroline V. Ketron, and Michael R. Waters, pp. 293-309. Texas A\&M University Press, College Station.

He, Feng J., and Graham A. MacGregor

2008 Beneficial Effects of Potassium on Human Health. Physiologia Plantarum 133(4):725-735.

Heizer, Robert Fleming, and Martin A. Baumhoff

1970 Big Game Hunters in the Great Basin: A Critical Review of the Evidence. In Papers on the Anthropology of the Western Great Basin, pp. 1-12. Contributions of the University of California Research Facility No. 7. University of California, Berkeley.

Helzer, Margaret M.

2001 Paleoethnobotany and Household Archaeology at the Bergen Site: A Middle Holocene Occupation in the Fort Rock Basin, Oregon. PhD dissertation, Department of Anthropology, University of Oregon, Eugene.

Herzog, Nicole M., and Anne T. Lawlor

2016 Reevaluating Diet and Technology in the Archaic Great Basin Using Starch Grain Assemblages from Hogup Cave, Utah. American Antiquity 81:664-681.

Hill, Matthew E.

2008 Variation in Paleoindian Fauna Use on the Great Plains and Rocky Mountains of North America. Quaternary International 191:34-52.

Hitchcock, C. Leo, and Arthur Cronquist

2018 Flora of the Pacific Northwest: An Illustrated Manual. 2nd edition. University of Washington Press, Seattle.

Hockett, Bryan

2007 Nutritional Ecology of Late Pleistocene to Middle Holocene Subsistence in the Great Basin: Zooarchaeological Evidence from Bonneville Estates Rockshelter. In Paleoindian or Paleoarchaic? Great Basin Human Ecology at the Pleistocene-Holocene Transition, edited by Kelly E. Graf and Dave N. Schmitt, pp. 204-230. University of Utah Press, Salt Lake City.

2012a Optimizing Energy-The Epistemology of Primitive Economic Man. In Archaeology: New Approaches in Theory and Techniques, edited by Imma Ollich-Castanyer, pp. 3-40. IntechOpen, Rijeka, Croatia.

2012b The Consequences of Middle Paleolithic Diets on Pregnant Neanderthal Women. Quaternary International 264:78-82.

Hockett, Bryan S., and Jonathan A. Haws

2003 Nutritional Ecology and Diachronic Trends in Paleolithic Diet and Health. Evolutionary Anthropology 5:211-216

2005 Nutritional Ecology and the Human Demography of Neandertal Extinction. Quaternary International 137:21-34

Hollenbach, Kandace D.

2007 Gathering in the Late Paleoindian Period: Archaeobotanical Remains from Dust Cave, Alabama. In Foragers of the Terminal Pleistocene in North America edited by Renee B. Walker and Boyce N. Driskell, pp. 132-147. University of Nebraska Press, Lincoln.

Hurst, Stance, Brian J. Carter, and Nancy Beavan Athfield

2010 Investigation of a 10,214 Year Old Late Paleoindian Bison Kill at the Howard Gully Site in Southwestern Oklahoma. Plains Anthropologist 55:25-37.

Irwin, Ann M., and Ula Laura Moody

1978 The Lind Coulee Site (45GR97). Project Report No. 56. Washington Archaeological Research Center, Washington State University, Pullman.

Janetski, Joel C., Mark L. Bodily, Bradley A. Newbold, and David T. Yoder

2012 The Paleoarchaic to Early Archaic Transition to the Colorado Plateau: The Archaeology of North Creek Shelter. American Antiquity 77:125-159.

Jazwa, Christopher S., Geoffrey M. Smith, Richard L. Rosencrance, Daron G. Duke, and Dan Stueber

2021 Reassessing the Radiocarbon Date from the Buhl Burial from South-Central Idaho and Its Relevance to the Western Stemmed Tradition-Clovis Debate in the Intermountain West. American Antiquity 86:173-182. 
Jenkins, Dennis L.

2004 The Grasshopper and the Ant: Middle Holocene Occupations and Storage Behavior at the Bowling Dune Site in the Fort Rock Basin, Central Oregon. In Early and Middle Holocene Archaeology of the Northern Great Basin, edited by Dennis L. Jenkins, Thomas J. Connolly, and C. Melvin Aikens, pp. 213-155. Anthropological Papers 62. University of Oregon Museum of Natural History, Eugene.

Jenkins, Dennis L., Loren G. Davis, Thomas W. Stafford, Paula F. Campos, Bryan S. Hockett, George T. Jones, Linda Scott Cummings, Chad Yost, Thomas J. Connolly, and Robert M. Yohe

2012 Clovis age Western Stemmed Projectile Points and Human Coprolites at the Paisley Caves. Science 337 (6091):223-228.

Jenkins, Dennis L., Justin A. Holcomb, and Katelyn N. McDonough

2017 Current Research at the Connley Caves (35LK50): Late Pleistocene/Early Holocene Western Stemmed Tradition Occupations in the Fort Rock Basin, Oregon. PaleoAmerica 3:188-192.

Jennings, Jesse D.

1957 Danger Cave. University of Utah Anthropological Papers 27. University of Utah Press, Salt Lake City.

Kelly, Isabel Truesdell

1932 Ethnography of the Surprise Valley Paiute. In University of California Publications in American Archaeology and Ethnology Volume XXXI, edited by A. L. Kroeber, Robert H. Lowie, and Ronald L. Olson, pp. 67-210. University of California Press, Berkeley.

Kelly, Robert L.

2013 The Lifeways of Hunter-Gatherers: The Foraging Spectrum. Cambridge University Press, Cambridge.

Kelly, Robert L., and Lawrence C. Todd

1988 Coming into the Country: Early Paleoindian Hunting and Mobility. American Antiquity 53:231-244.

Kennedy, Jaime L.

2018 A Paleoethnobotanical Approach to 14,000 Years of Great Basin Prehistory: Assessing Human-Environmental Interactions through the Analysis of Archaeological Plant Data at Two Oregon Rockshelters. PhD dissertation, Department of Anthropology, University of Oregon, Eugene.

Kennedy, Jaime L., and Geoffrey M. Smith

2016 Paleoethnobotany at the LSP-1 Rockshelter, South Central Oregon: Assessing the Nutritional Diversity of Plant Foods in Holocene Diet. Journal of Archaeological Science: Reports 5:640-648.

Kilby, J. David, Sean P. Farrell, and Marcus J. Hamilton

2021 New Investigations at Bonfire Shelter, Texas Examine Controversial Bison Jumps and Bone Beds. Plains Anthropologist 66:34-57.

Laires, Maria José, Cristina Paula Monteiro, and Manuel Bicho 2004 Role of Cellular Magnesium in Health and Human Disease. Frontiers in Bioscience 9:262-276.

Lee, Gyoung-Ah

2012 Taphonomy and Sample Size Estimation in Paleoethnobotany. Journal of Archaeological Science 39:648-655.

Lothrop, Jonathan C., Darrin L. Lowery, Arthur E. Spiess, and Christopher J. Ellis

2016 Early Human Settlement of Northeastern North America. PaleoAmerica 2:192-251.

Louderback, Lisbeth A.

2014 The Ecology of Human Diets during the Holocene at
North Creek Shelter, Utah. PhD dissertation, Department of Anthropology, University of Washington, Seattle.

Louderback, Lisbeth A., and Bruce M. Pavlik

2017 Starch Granule Evidence for the Earliest Potato Use in North America. PNAS 114:7606-7610.

Mackie, Madeline E., Todd A. Surovell, Matthew O'Brien, Robert L. Kelly, Spencer Pelton, C. Vance Haynes, George C. Frison, et al.

2020 Confirming a Cultural Association at the La Prele Mammoth Site (48CO1401), Converse County, Wyoming. American Antiquity 85:554-572.

Madsen, David B.

2015 A Framework for the Initial Occupation of the Americas. PaleoAmerica 1:217-250.

Mahar, James Michael

1953 Ethnobotany of the Oregon Paiutes of the Warm Springs Indian Reservation. Bachelor's thesis, Reed College, Portland, Oregon.

Mailer, Gideon, and Nicola Hale

2013 Decolonizing the Diet: Synthesizing Native-American History, Immunology, and Nutritional Science. Journal of Evolution and Health 1(1):1-40.

Martin, Paul S.

1973 The Discovery of America. Science 179:969-974.

McDonough, Katelyn N.

2019 Middle Holocene Menus: Dietary Reconstruction from Coprolites at the Connley Caves, Oregon, USA. Archaeological and Anthropological Sciences 11:5963-5982.

McDonough, Katelyn, Richard L. Rosencrance, Justin Holcomb, and Dennis L. Jenkins

2018 Update from the 2017 and 2018 Excavations by the University of Oregon Archaeology Field School at Connley Caves 2 and 5 (35LK50), Fort Rock Basin, Oregon. Current Archaeological Happenings in Oregon 43(3):3-8.

Meltzer, David J.

2020 Overkill, Glacial History, and the Extinction of North America's Ice Age Megafauna. PNAS 117:28555-28563.

Mentzer, Susan M.

2014 Microarchaeological Approaches to the Identification and Interpretation of Combustion Features in Prehistoric Archaeological Sites. Journal of Archaeological Method and Theory 21:616-668.

Moerman, Daniel E.

1998 Native American Ethnobotany. Timber Press, Portland, Oregon.

Morehouse, Nathan I., David Raubenheimer, Adam Kay, and Susan M. Bertram

2020 Integrating Nutritional and Behavioral Ecology: Mutual Benefits and New Frontiers. Advances in the Study of Behavior 52:29-63.

Murphey, Edith Van Allen

1959 Indian Uses of Native Plants. Meyerbooks, Glenwood, Ohio.

O'Connell, James F., Kevin T. Jones, and Steven R. Simms

1982 Some Thoughts on Prehistoric Archaeology in the Great Basin. In Man and Environment in the Great Basin, edited by David B. Madsen and James F. O'Connell, pp. 227-240. Society for American Archaeology, Washington, DC.

Park, Willard Z., and Catherine S. Fowler

1989 Willard Z. Park's Ethnographic Notes on the Northern Paiute of Western Nevada, 1933-1940. Anthropological Papers 114. University of Utah Press, Salt Lake City. 
Phillips, Katherine M., Pamela R. Pehrsson, Wanda W. Agnew, Angela J. Scheett, Jennifer R. Follett, Henry C. Lukaski, and Kristine Y. Patterson

2014 Nutrient Composition of Selected Traditional United States Northern Plains Native American Plant Foods. Journal of Food Composition and Analysis 34:136-152.

Pinson, Ariane Oberling

1999 Foraging in Uncertain Times: The Effects of Risk on Subsistence Behavior during the Pleistocene-Holocene Transition in the Oregon Great Basin. PhD dissertation, Department of Anthropology, University of New Mexico, Albuquerque.

Prouty, Guy L.

1994 Root Crop Exploitation and the Development of Upland Habitation Sites: A Prospectus for Paleoethnobotanical and Archaeological Research into the Distribution of Economic Plants in the Fort Rock Basin. In Archaeological Research in the Northern Great Basin: Fort Rock Archaeology since Cressman, edited by C. Melvin Aikens and Dennis L. Jenkins, pp. 573-598. Archaeological Papers 50. Museum of Natural History and Department of Anthropology, University of Oregon, Eugene.

2004 Plants and Prehistory: Paleoethnobotanical Investigations in the Fort Rock Basin Lowlands. In Early and Middle Holocene Archaeology of the Northern Great Basin, edited by Dennis L. Jenkins, Thomas J. Connolly, and C. Melvin Aikens, pp. 157-166. Anthropological Papers 62. Museum of Natural History and Department of Anthropology, University of Oregon, Eugene.

Puseman, Kathryn, and Chad Yost

2011 Macrofloral Analysis of Samples from the Dirty Shame Rockshelter (35ML65), Malheur County, Oregon. Technical Report 11-065. PaleoResearch Institute, Golden, Colorado.

$\mathrm{R}$ Core Team

2020 R: A Language and Environment for Statistical Computing. R Foundation for Statistical Computing, Vienna, Austria. https://www.R-project.org/.

Ramakrishnan, Usha, Renu Manjrekar, Juan Rivera, Teresa Gonzáles-Cossío, and Reynaldo Martorell

1999 Micronutrients and Pregnancy Outcome: A Review of the Literature. Nutrition Research 19:103-159.

Raubenheimer, David, and Stephen J. Simpson

1993 The Geometry of Compensatory Feeding in the Locust. Animal Behaviour 45:953-964.

Raubenheimer, David, and Stephen J. Simpson

1999 Integrating Nutrition: A Geometrical Approach. Entomologia Experimentalis et Applicata 91:67-82.

2016 Nutritional Ecology and Human Health. Annual Review of Nutrition 36:603-626.

2018 Nutritional Ecology and Foraging Theory. Current Opinion in Insect Science 27:38-45.

Ray, Verne Frederick

1963 Primitive Pragmatists: The Modoc Indians of Northern California. University of Washington Press, Seattle.

Rhode, David

1999 The Role of Paleoecology in the Development of Great Basin Anthropology Today. In Models for the Millennium: Great Basin Anthropology Today, edited by Charlotte Beck, pp. 29-49. University of Utah Press, Salt Lake City.
2003 Coprolites from Hidden Cave, Revisited: Evidence for Site Occupation History, Diet and Sex of Occupants. Journal of Archaeological Science 30:909-922.

Rhode, David, and Lisbeth A. Louderback

2007 Dietary Plant Use in the Bonneville Basin during the Terminal Pleistocene/Early Holocene Transition. In Paleoindian or Paleoarchaic? Great Basin Human Ecology at the Pleistocene/Holocene Transition, edited by Kelly E. Graf and Dave N. Schmitt, pp. 231-247. University of Utah Press, Salt Lake City.

Rhode, David, David B. Madsen, and Kevin T. Jones

2006 Antiquity of Early Holocene Small-Seed Consumption and Processing at Danger Cave. Antiquity 80:328-339.

Rosencrance, Richard L.

2019 Assessing the Chronological Variation within Western Stemmed Tradition Projectile Points. Master's thesis, Department of Anthropology, University of Nevada, Reno.

Rosenthal, Jeffrey S., and Richard T. Fitzgerald

2012 The Paleo-Archaic Transition in Western California. In From the Pleistocene to the Holocene: Human Organization and Cultural Transformations in Prehistoric North America, edited by C. Britt Bousman and Bradley J. Vierra. pp. 67-103. Texas A\&M University Press, College Station.

Sanford, Patricia Ruth

1983 An Analysis of Megascopic Plant remains and Pollen from Dirty Shame Rockshelter, Southeastern Oregon. $\mathrm{PhD}$ dissertation, Department of Anthropology, University of Oregon, Eugene.

Schmitt, Dave N., and David B. Madsen

2005 Camels Back Cave. Anthropological Papers 125. University of Utah Press, Salt Lake City.

Sellards, Elias H.

1952 Early Man in North America. Texas Memorial Museum, Austin.

Shillito, Lisa-Marie, Helen L. Whelton, John C. Blong, Dennis L. Jenkins, Thomas J. Connolly, and Ian D. Bull

2020 Pre-Clovis Occupation of the Americas Identified by Human Faecal Biomarkers in Coprolites from Paisley Caves, Oregon. Science Advances 6(29):eaba6404.

Simms, Steven R.

1985 Acquisition Cost and Nutritional Data on Great Basin Resources. Journal of California and Great Basin Anthropology 7:117-126.

1987 Behavioral Ecology and Hunter-Gatherer Foraging: An Example from the Great Basin. BAR International Series 381. British Archaeological Reports, Oxford.

Simpson, Stephen J., and David Raubenheimer

1993 A Multi-Level Analysis of Feeding Behaviour: The Geometry of Nutritional Decisions. Philosophical Transactions of the Royal Society of London, Series B: Biological Sciences 342:381-402.

2012 The Nature of Nutrition. Princeton University Press, Princeton, New Jersey.

Smith, Geoffrey M., and Pat Barker

2017 The Terminal Pleistocene/Early Holocene Record in the Northwestern Great Basin: What We Know, What We Don't Know, and How We May Be Wrong. PaleoAmerica 3:13-47.

Smith, Geoffrey M., Daron Duke, Dennis L. Jenkins, Ted Goebel, Loren G. Davis, Patrick O'Grady, Dan Stueber, Jordan E. Pratt, and Heather L. Smith

2020 The Western Stemmed Tradition: Problems and 
Prospects in Paleoindian Archaeology in the Intermountain West. PaleoAmerica 6:23-42.

Spier, Leslie

1930 Klamath Ethnography. Publications in American Archaeology and Ethnology Vol. 30. University of California Press, Berkeley.

Stenholm, Nancy A.

1994 Paleoethnobotanical Analysis of Archaeological Samples Recovered in the Fort Rock Basin. In Archaeological Research in the Northern Great Basin: Fort Rock Archaeology since Cressman, edited by C. Melvin Aikens and Dennis L. Jenkins, pp. 531-560. Archaeological Papers 50. Department of Anthropology and State Museum of Anthropology, University of Oregon, Eugene.

Stevenson, Matilda Coxe

1915 Ethnobotany of the Zuni Indians. US Government Printing Office, Washington DC.

Steward, Julian

1933 Ethnography of the Owens Valley Paiute. University of California Publications in American Archaeology and Ethnology 33(3):233-250.

Surovell, Todd A., and Nicole M. Waguespack

2009 Human Prey Choice in the Late Pleistocene and Its Relation to Megafaunal Extinctions. In American Megafaunal Extinctions at the End of the Pleistocene, edited by Gary Haynes, pp. 77-105. Springer, New York.

Testart, Alain, Richard G. Forbis, Brian Hayden, Tim Ingold, Stephen M. Perlman, David L. Pokotylo, Peter RowleyConwy, and David E. Stuart

1982 The Significance of Food Storage among HunterGatherers: Residence Patterns, Population Densities, and Social Inequalities. Current Anthropology 23:523-537.

Train, Percy, James R. Henrichs, and W. Andrew Archer

1941 Medicinal Uses of Plants by Indian Tribes of $\mathrm{Ne}$ vada. US Department of Agriculture, Washington, DC.

USDA (United States Department of Agriculture Agricultural Research Service)

2019 FoodData Central. https://fdc.nal.usda.gov, accessed March 12, 2021.
Waguespack, Nicole M.

2013 Pleistocene Extinctions: The State of Evidence and the Structure of Debate. In Paleoamerican Odyssey, edited by Kelly E. Graf, Caroline V. Ketron, and Michael R. Waters, pp. 311-319. Texas A\&M University Press, College Station.

Walker, Mike, Martin J. Head, John Lowe, Max Berkelhammer, Svante Björck, Hai Cheng, Les C. Cwynar, et al.

2019 Subdividing the Holocene Series/Epoch: Formalization of Stages/Ages and Subseries/Subepochs, and Designation of GSSPs and Auxiliary Stratotypes. Journal of Quaternary Science 34:173-186.

Winterhalder, Bruce, and Eric Alden Smith

2000 Analyzing Adaptive Strategies: Human Behavioral Ecology at Twenty-Five. Evolutionary Anthropology: Issues News and Reviews 9(2):51-72.

Wolfe, Allison L., and Jack M. Broughton

2020 A Foraging Theory Perspective on the Associational Critique of North American Pleistocene Overkill. Journal of Archaeological Science 119:105162.

Yoder, David T., Mark L. Bodily, Sara Hill, Joel C. Janetski, and Bradley A. Newbold

2010 The Onset of Small Seed Processing on the Colorado Plateau. Kiva 75:425-446.

Zeanah, David W., and Steven R. Simms

1999 Modeling the Gastric: Great Basin Subsistence Studies since 1982 and the Evolution of General Theory. In Models for the Millennium: Great Basin Anthropology Today, edited by Charlotte Beck, pp. 118-140. University of Utah Press, Salt Lake City.

Zigmond, Maurice L.

1981 Kawaiisu Ethnobotany. University of Utah Press, Salt Lake City.

Submitted March 31, 2021; Revised July 18, 2021; Accepted October 3, 2021 